\title{
التزامن في اللغة العربية (دراسة دلالية)
}

\section{فؤاد رمضان محمد حمادة}

أستاذ مشارك- جامعة القدس المفتوحة- فلسطين

foadhamada@gmail.com 


$$
\text { المجلة الدولية للدراسـات اللغوية والأدبية العربية }
$$

International Journal for Arabic Linguistics and Literature Studies (JALLS)

\section{www.refaad.com}

Journal Homepage: https://www.refaad.com/views/JALS/home.aspx ISSN: 2663-5860(Online) 2663-5852(Print)

\title{
التزامن في اللغة العربية (دراسة دلالية)
}

\author{
فؤاد رمضيان محمد حمادة \\ أستاذ مشارك- جامعة القدس المفتوحة- فلسطين \\ foadhamada@gmail.com
}

DOI: https://doi.org/10.31559/JALLS2021.3.2.3 2021/6/19 2021/5/18 مراجعة البحث: 2021/6/7 قبول البحث البحث:

\section{الملخخ:}

كثر استعمال التزامن في العلوم الحديثة لغة واصطلاحاً، بما يوجب تأصيل معناه، وكيفية استعماله. فقد أغفلت معاجمنا

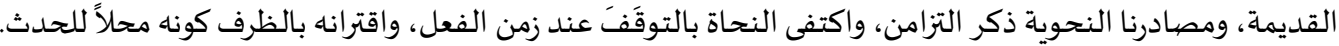

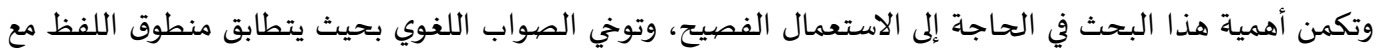
مدلوله، ومعرفة التراكيب اللغوية التي تعبر عن القصديد.

وتهدف هذه الدراسة إلى التعرف على الأدوات، والتراكيب، والأساليب اللغوية التي استخدمتها العرب للدلالة على التزامن، والاستفادة منها في الاستخدام المعاصر بما يضمن سلامة اللغة.

واعتمد الباحث المنهج الوصفي والمنهج الاستقرائي، حيث تتبع الباحث الشواهد اللغوية من القرآن الكريم وكلام العرب الماري

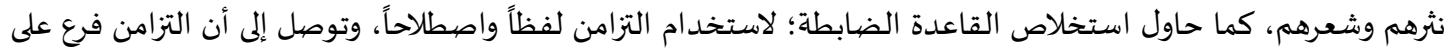

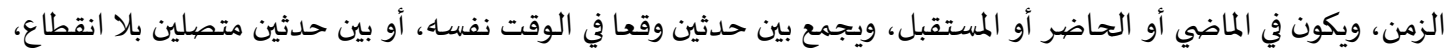
ويعبر عنه بصيغة صرفية أو تركيب نحوي، أو قرينة لفظية. الكلمات المفتاحية: التزامن؛ حدثين؛ وقت واحد؛ صيغة صرفية؛ تركيب نحوي؛ قرينة لفظية.

مقدمة الدراسة: توقفت عناية النحاة بالزمن عند الظروف باعتبارها محلاً للحدث. قال الأنباري (1999، 141):" فإن قيل: فَلِمَ سُمّيّ ظرفًا؟ قيل:

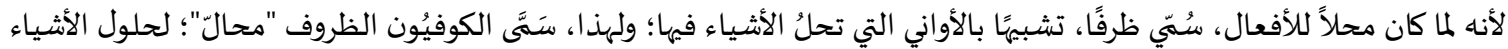

أما التزامن، فلم يرد ذكره في معاجمنا اللغوية القديمة، ولم يبوب له النحاة، ولم يفرد بدراسة مستقلة، - فيما أعلم- وبقيت موضوعاته مشتتاة في كتب النحو، دون التصريح بالتزامن.

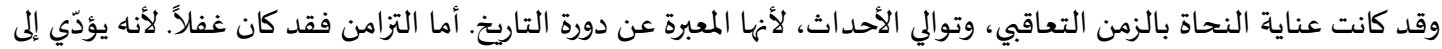

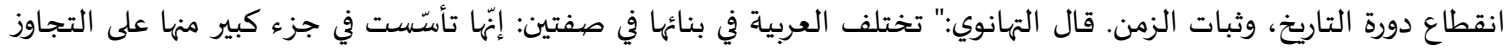

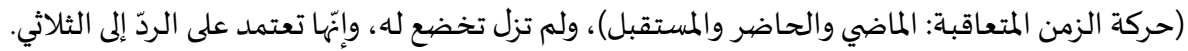

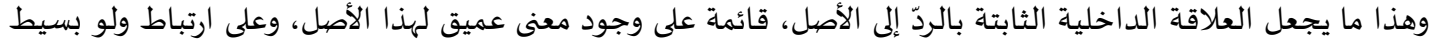

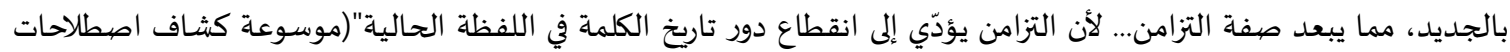

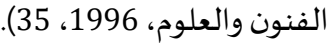


ولا شك أن اللغة أوسع من قواعد النحو وأمثلة النحاة، وأن أدوات التزامن وتركيبه وأساليبه متوفرة في اللغة، وأنه محتَمل

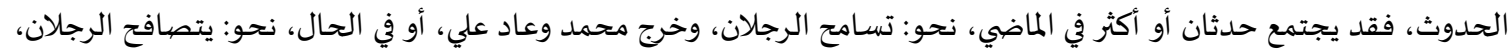

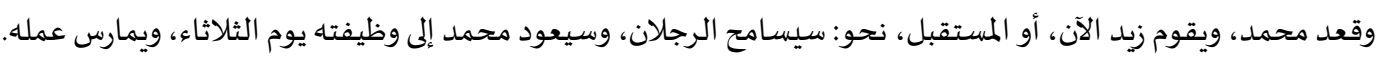

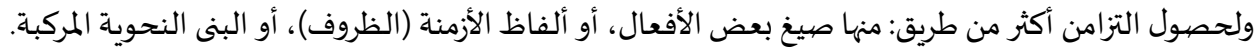

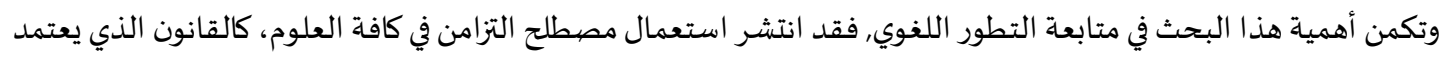

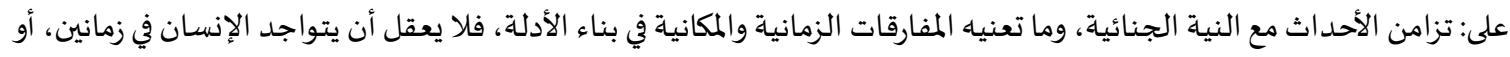

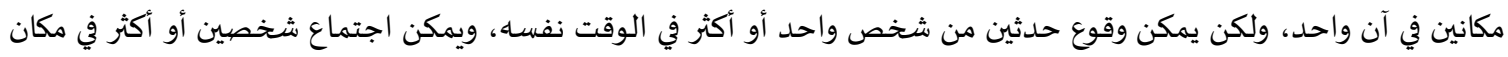

كما ظهر مع الأعمال العسكرية: كتزامن القصف الجوي مع الغزو البري والبحري، وظهر مع الخيارات السياسية: كتزامن

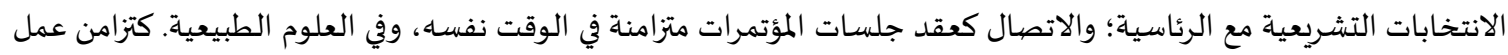
الأجهزة، وفي غيرها من العلوم مما يصعب حصره. وتهدف هذه الدراسة إلى معرفة الأدوات، والتراكيب، والأساليب اللغوية التي تؤدي معنى التزامن مع توخي الصهواب اللغوي. بحيث يتطابق منطوق اللفظ مع مدلوله.

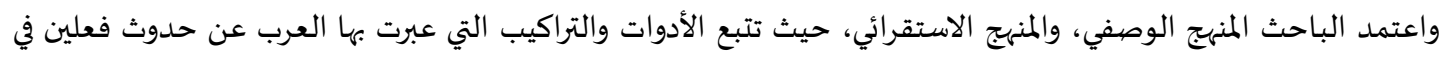

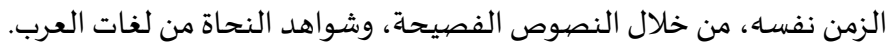

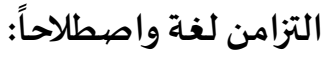

التزامن لغة: فرع على الزمن، والزَمَنُ اسم يدل على قليل الوقت وكثيره، ويُجمعُ على أَزمانِ، وأَزْهِنَة، وأَزْمُنْ (الجوهري، 1987 م،

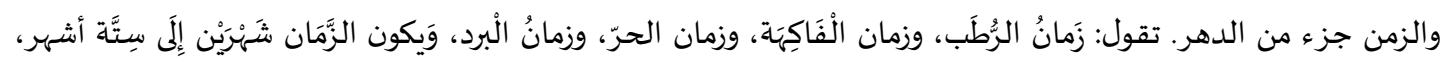

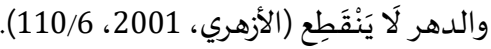

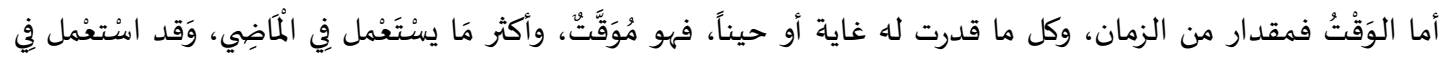

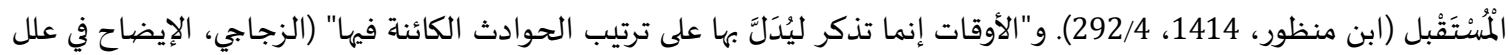
النحو، 1986، 115). (115). وقد تنوب بعض هذه الألفاظ (الزمن والوقت والدهر) في لغة العرب عن بعضها. من باب استعمال العام بدلاً من الخاص، أو

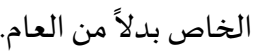
ودخل لفظ التزامن إلى المعاجم اللغوية المعاصرة من خلال تعريف عمر، أحمد مختار (2008، 997/2، 998) له في قوله: تزامنَ يتزامن، تزامُنًا، فهو مُتَزامِن.

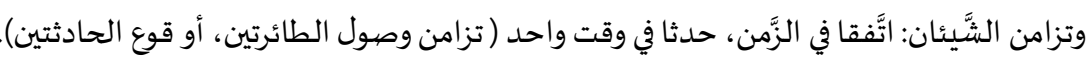

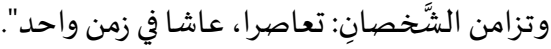

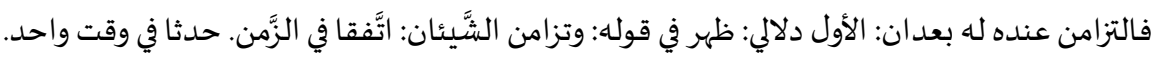

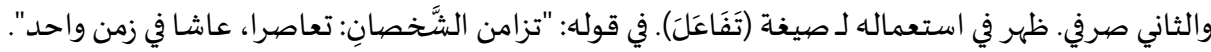

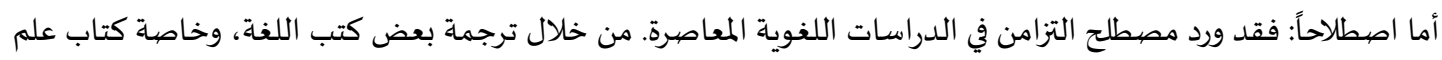

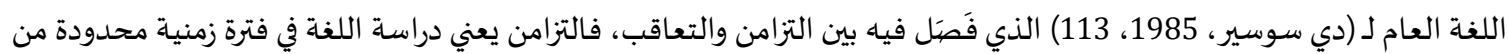

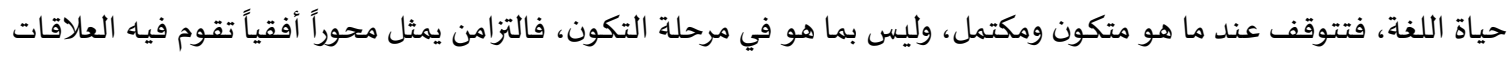
بين الأشياء المتواجدة على أساس ثابت ليس للزمان فيها أي دخل، أما التعاقب فيمثل محوراً عمودياً. تقوم فيه العلاقات بات بيات بين الأشياء

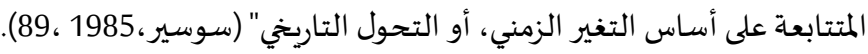

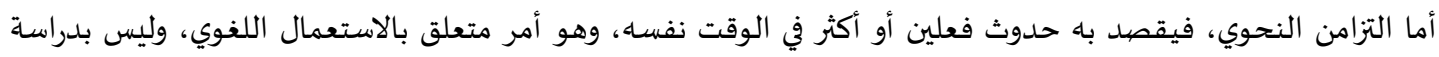

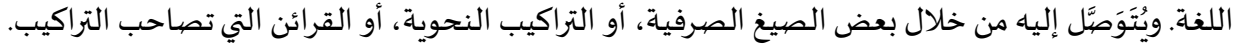


وأما المزامنة فهي ربط حدث بزمن كالتأجير بالشهر أو السنة: قال الكسائي: عاملته مُزامَنَةً من الزَمَنِ، كما يقال مشاهرةً من

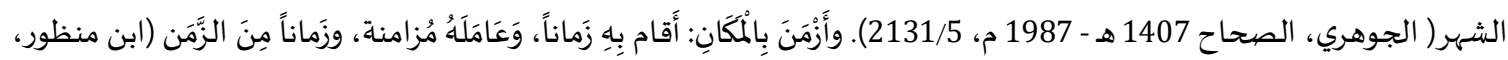

1414، ز م ن، 199/13).

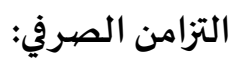

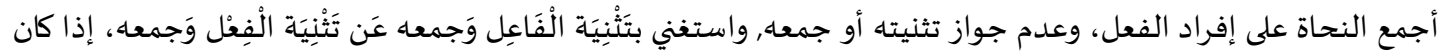

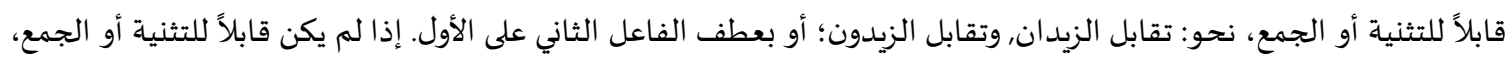

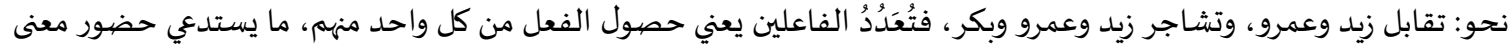

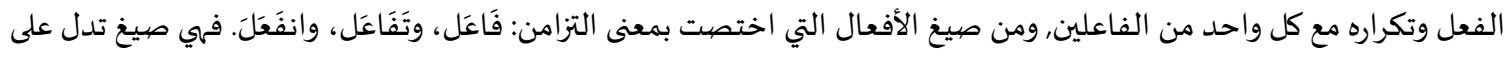

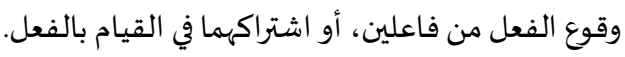

1

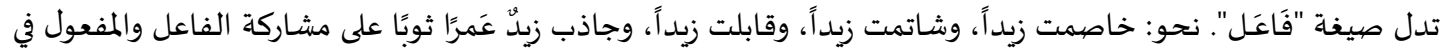

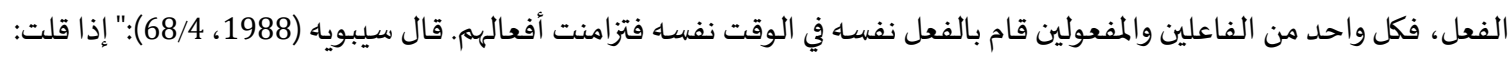

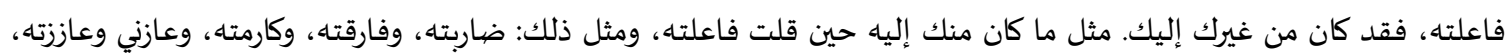

وخاصيمني وخاصمتهء".

وقد تأتي فَاعَل للدلالة على وقوع الفعل من الفاعل دون تشريك، نحو: سافر زيد، وناولت عمرًا الكتاب، وعاقب القاضي المتهمه.

2.

تأتي صيغة (تفاعل) للدلالة على مُشََارَكَةِة فاعلين فَصَّاعِدا في فعل واحد. نحو: نحو: تبادلنا الحديث، وتنافسنا، وتخاصهمنا،

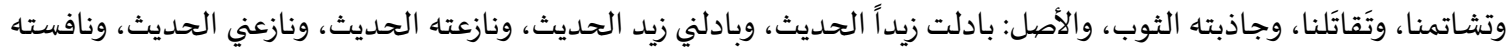

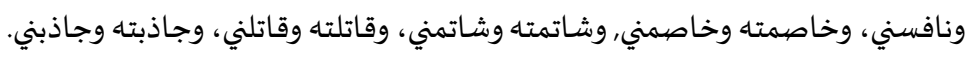

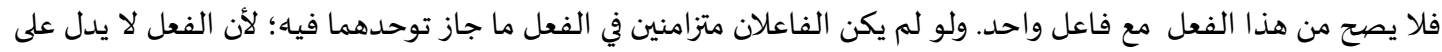
أكثر من زمن. وإذا لم يكن الفاعل قابلاً للتثنية والجمع جمع بين الفاعل والمفعول بالواو. نحو: تشارك زيد وعمرو، وتصالح سعيد وخالد,

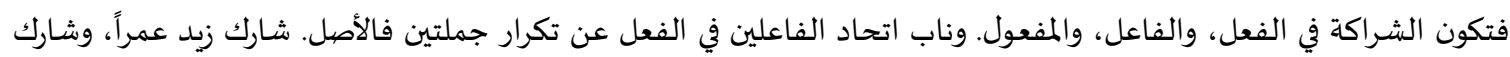

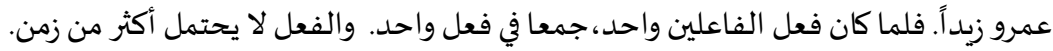

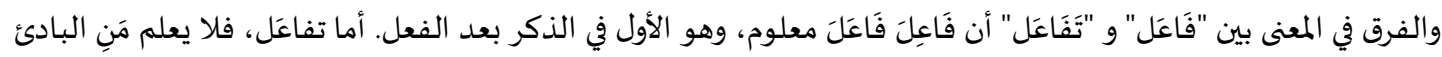

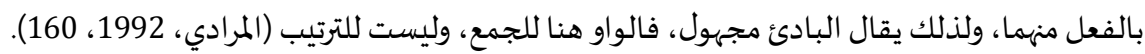

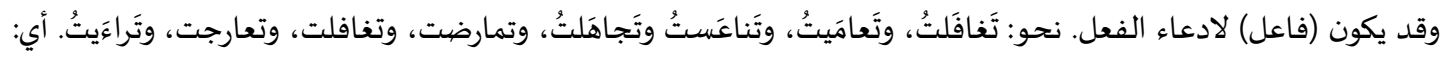

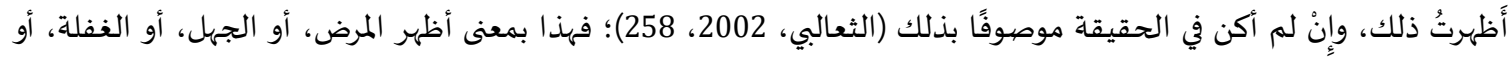
السُّكُر. وكل هذا يحكمها السياق.

3

تدل (افْتَعَل) على التشريك في الفعل، والفاعل، والمفعول، نحو: افترق الصديقان, واشترك زيد وبكر، والتقى الزيدان، واجتتمع

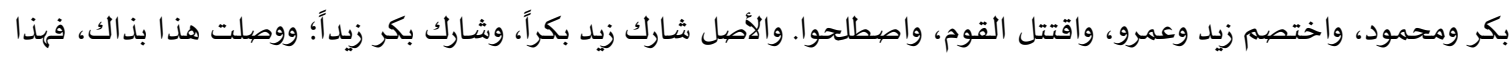

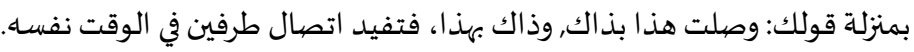

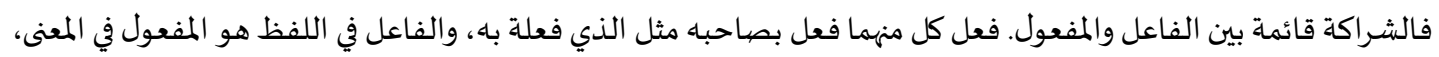
والعكس. واتّحدَ الفعلان في فعل واحد لاتفاقهما لفظاً ومعنى، والعطف على الفاعل. يدل على تكرار الفعل من المتعاطفين.

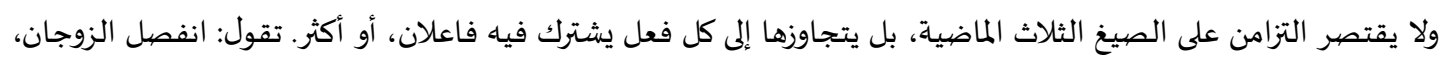

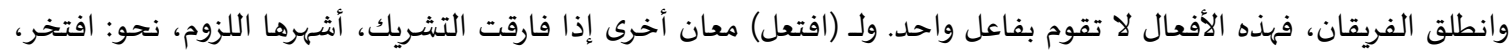
وارتفع، واطّلع. 


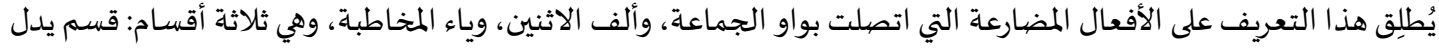

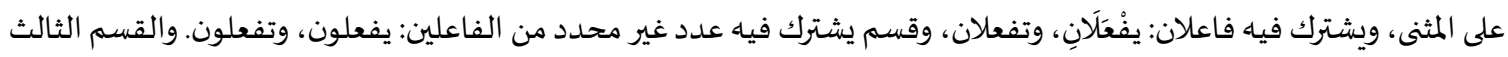
للمفردة المؤنثة.

فهذه الأفعال تدل على وقوع الفعل منهم جميعاً، وهي أفعال مضارعة تدل على الحال، ولا تتسع للمعاقبة. إلا أن تَرِدَ قرينة تصرف معنى هذه الأفعال عن الحال.

التزامن النحوي يعتمد التزامن النحوي على الإطار التركيبي الجامع لفعلين من الزمن نفسه، أو بين فعل، وما فيه معنى الفعل، كالصفات والمصادر.

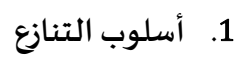

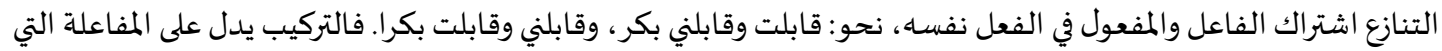

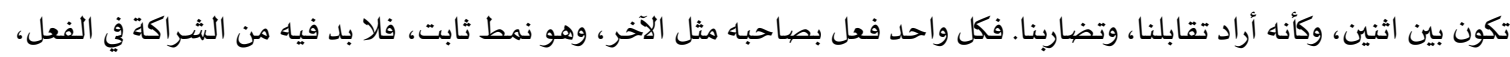
والعطف بالواو، والاستغناء بأحد المعمولين عن الآخر.

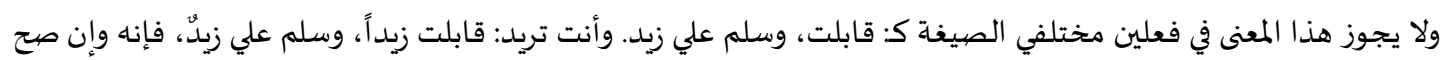

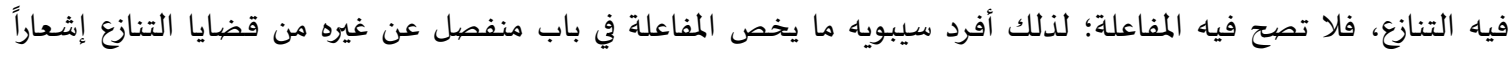

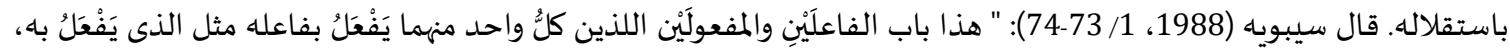

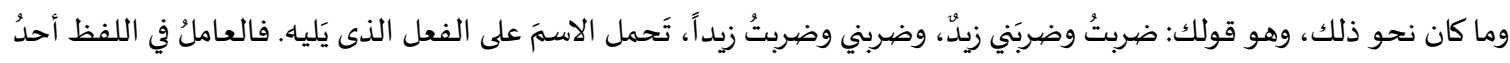

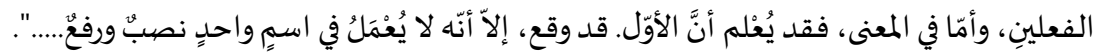

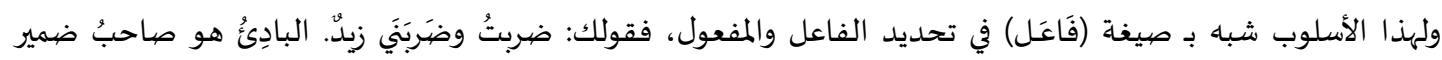

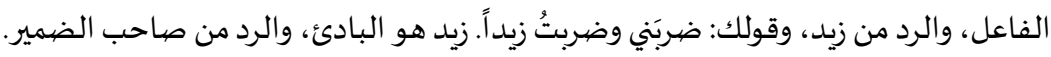

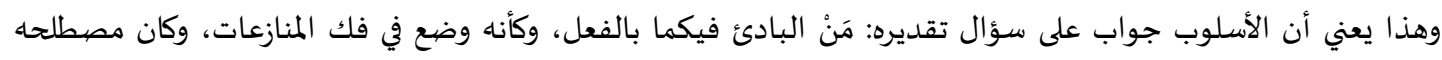
النحوي بمعناه اللغوي. وهذا الأسلوب كثير الاستعمال في التحقيقات القضائية؛ لأن البادئ بالفعل معتد، والثاني رادّ على الفعل، فهو بمنزلة المدافع عن نفسـا. 2. الجمع بين فعلي حاستين لفاعل واحد

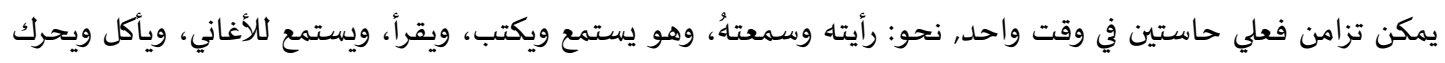

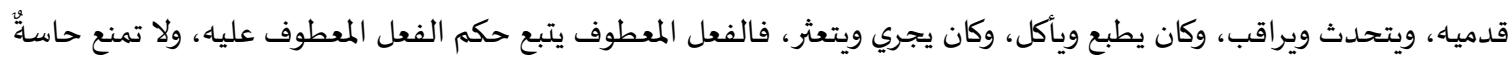

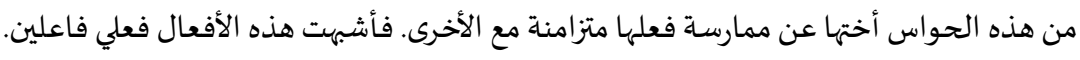

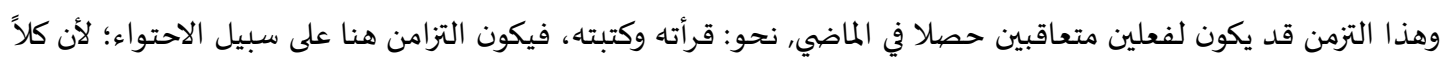

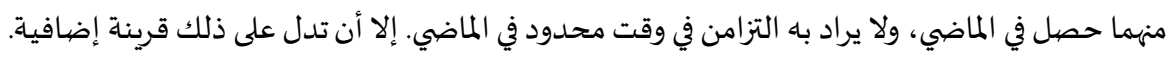

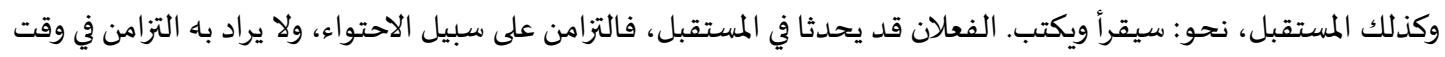
بعينه. وكذلك عطف الفعل المضارع على الفعل المضارع، نحو زيد يقرأ ويكتب، تكون الشراكة في الزمن المطلق إذا كانا خبرين، كأنك قلت: هو كاتب وقارئ، ويكون التزامن في الوقت نفسها إذا أردت بالواو الحال.

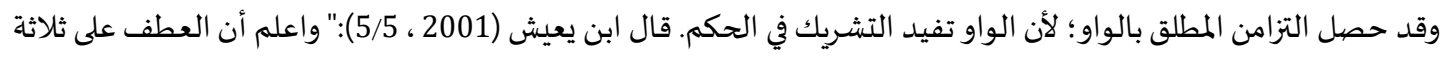

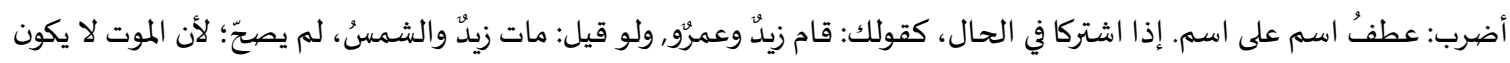

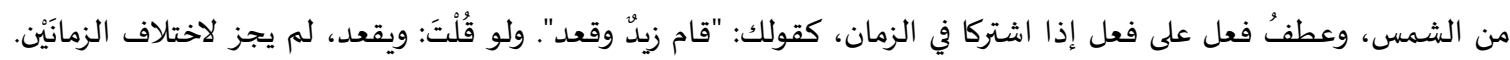

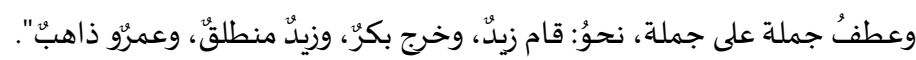




$$
\text { 3. أفعال القلوب وأخبارها }
$$

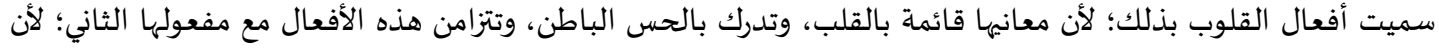

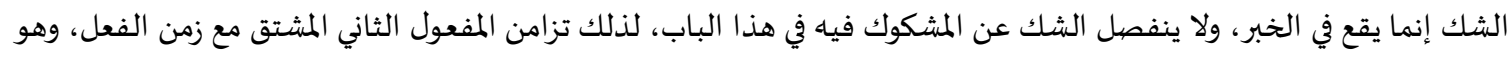

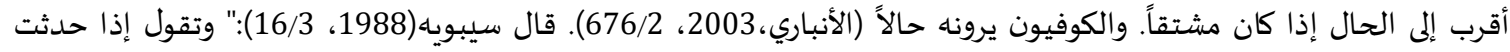

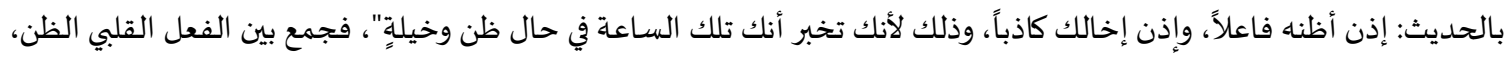

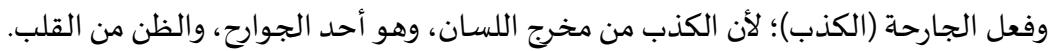
4. ألمعول لأجله

عدَّ النحاة تزامن المفعول لأجله مع الفعل شرطاً لصحة الأسلوب. قال ابن عقيل (1980، 186/2 ):" المفعول له: هو المصددر

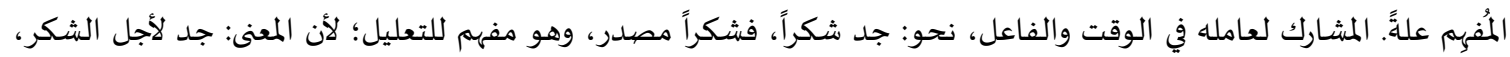

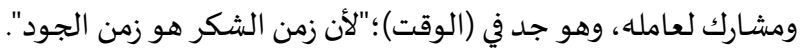

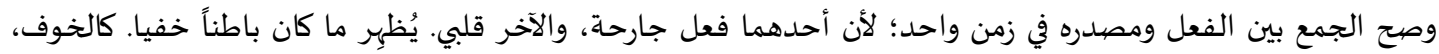

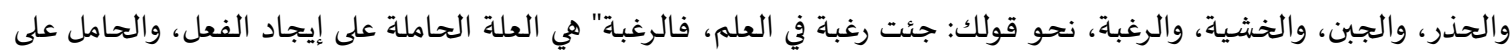

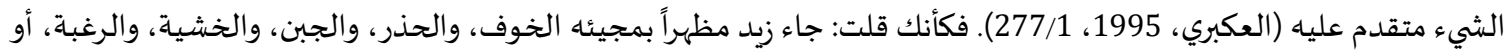
الحرص، وأشباه ذلك، "فهذه الأفعال الظاهرة تبدي لك البكان الباطنة.

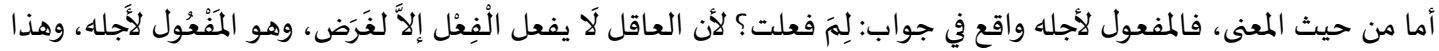

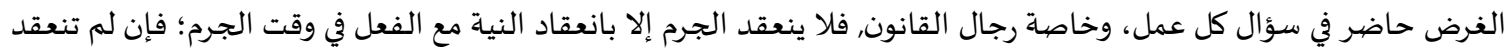

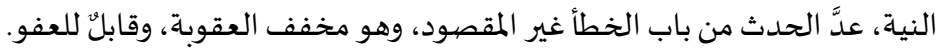

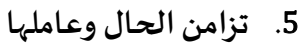

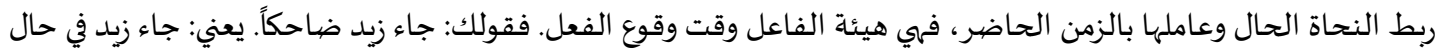

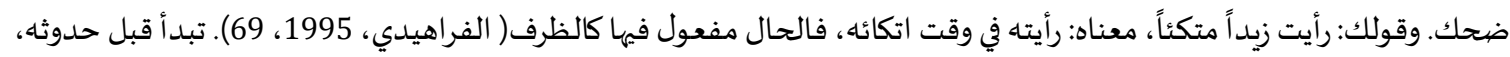

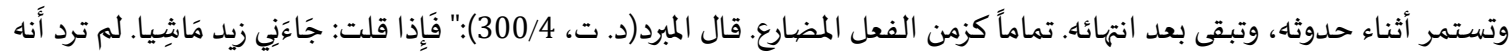

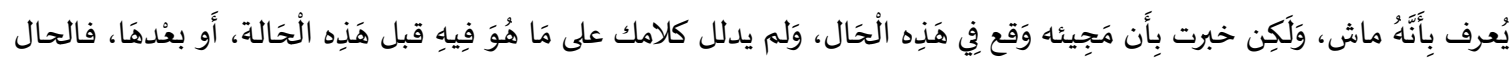

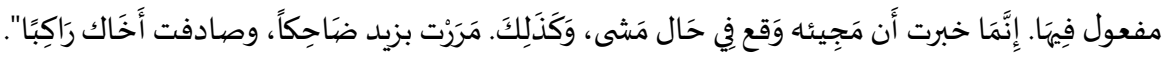

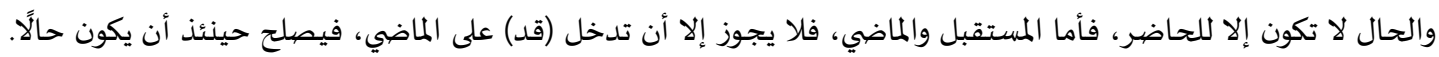

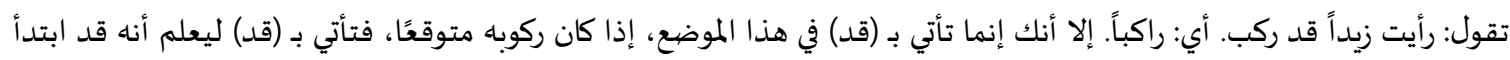

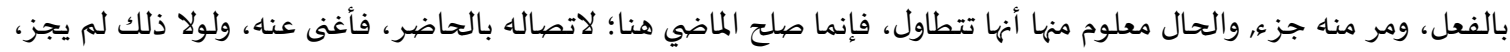

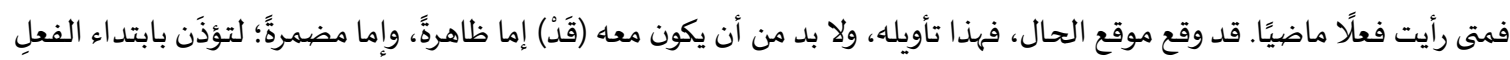

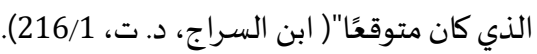

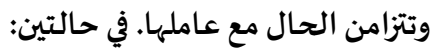
الأولى: تزامن الحال مع وقت النطق عامل بالعامل فها، وهذا يقتضي استعمال الفعل المضارع, فهو الفعل الذي يكون زمان الإخبار عن

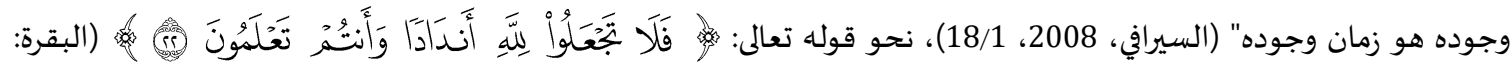

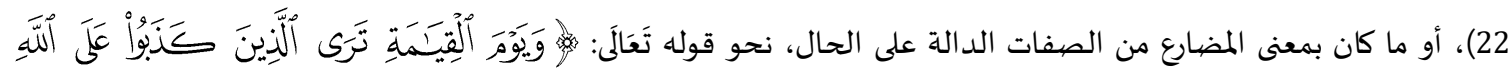

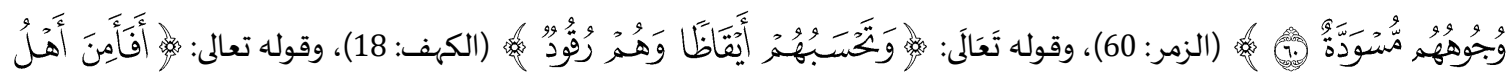

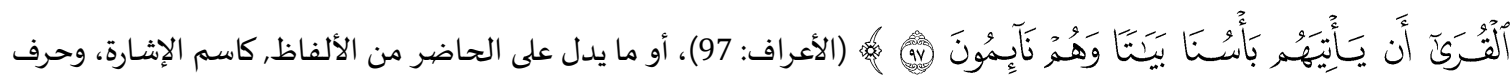

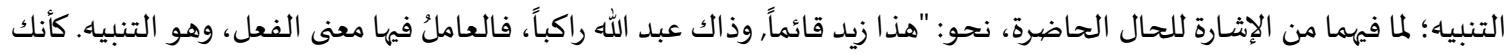

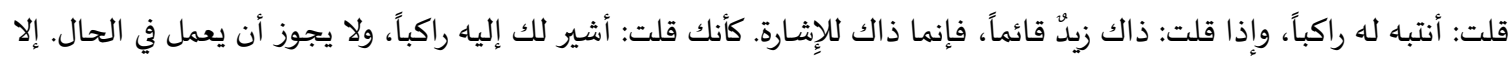
فعل، أو شيء في معنى الفعل؛ لأنها كالمفعول فيها. 
ولو قلت: زبد أخوك قائماً، وعبد الله أبوك ضاحكًا. كان غير جائز ، وذلك أنه ليس ههنا فعل, ولا معنى فعل، ولا يستقيم أن يكون

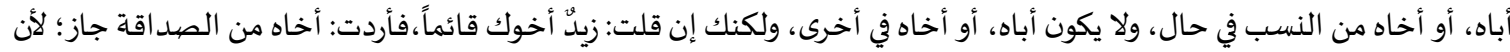

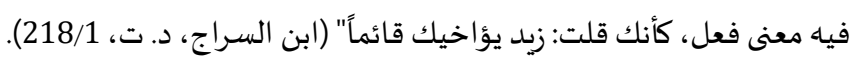

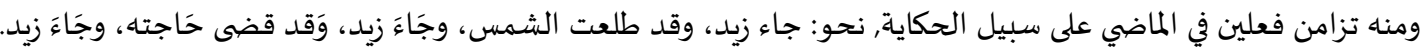

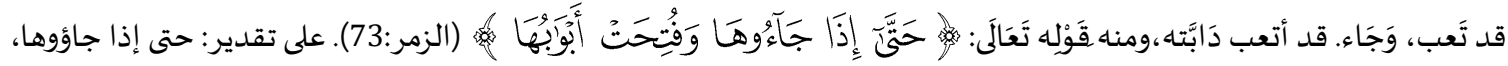

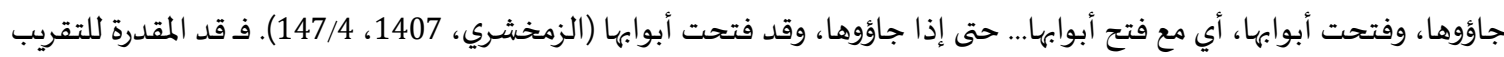
من الحال، والواو للحال. أي: جاءوها، وهذه حالها، فيتزامن وقت المجيء مع وقت فتح الأبواب.

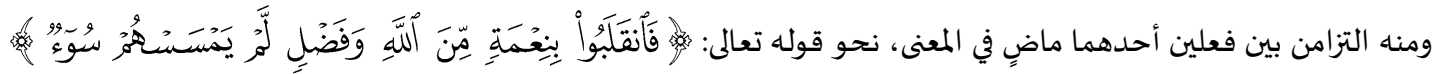

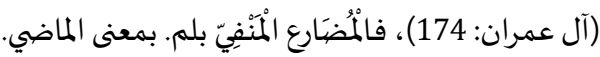

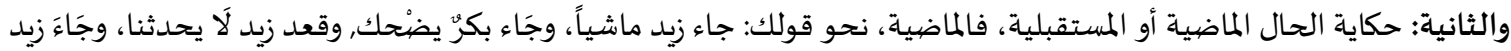

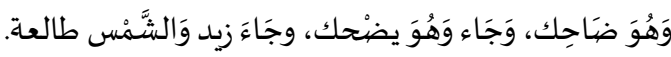

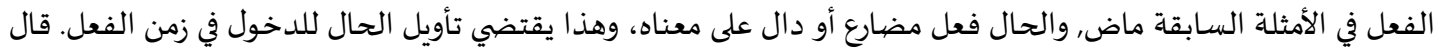

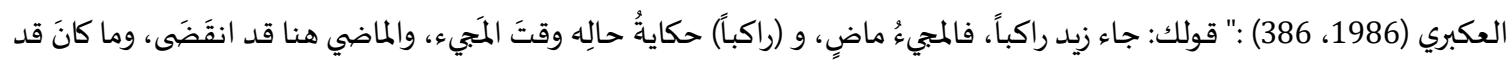

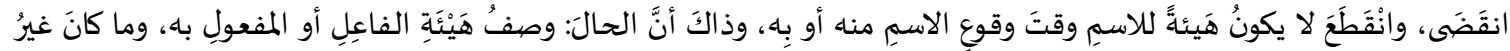

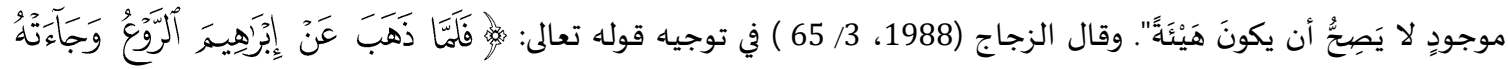

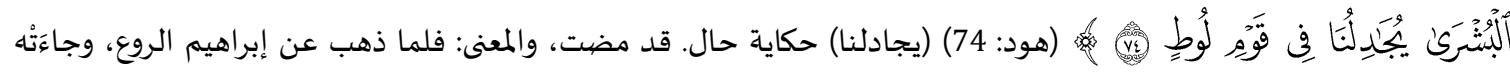

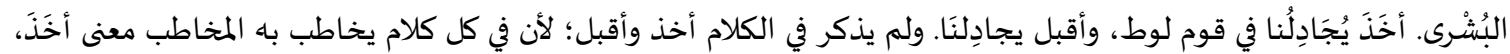

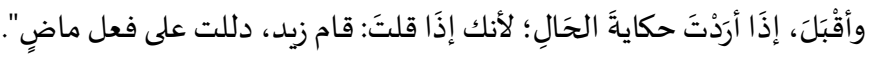

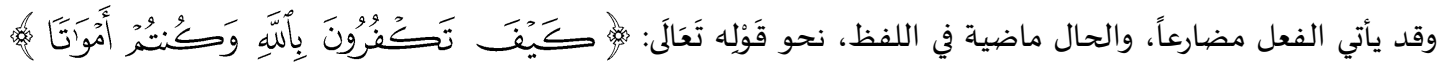
(البقرة: 28). فيتأول النحاة الحال من مضمون الجملة، وليس من منطوق اللفظ. قال الزمخشري(1407 هـ، 121/1):" الواو في قَوُلهِ

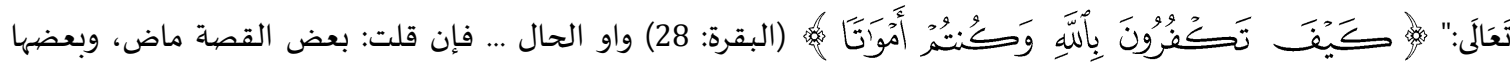

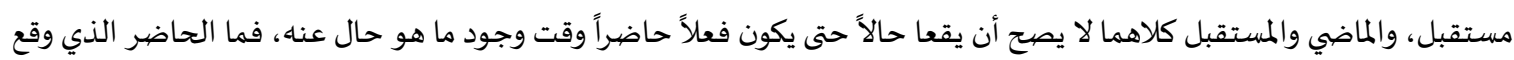

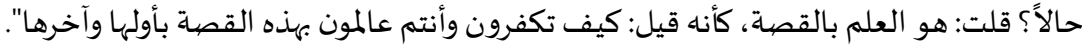

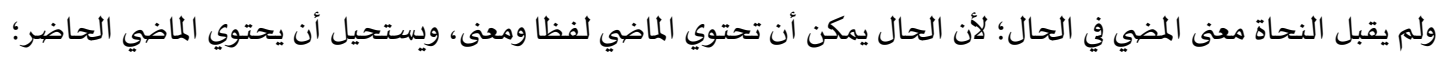

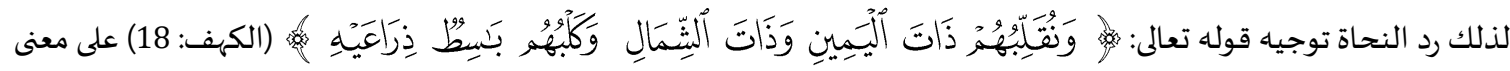

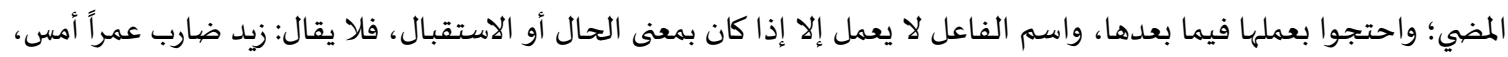

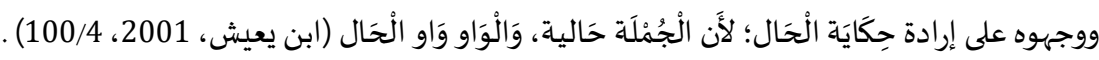

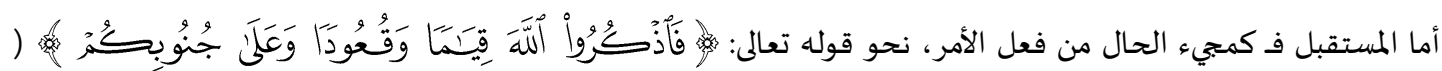
النساء: 103) فقياماً : حال، وقعوداً: حال ثانية.

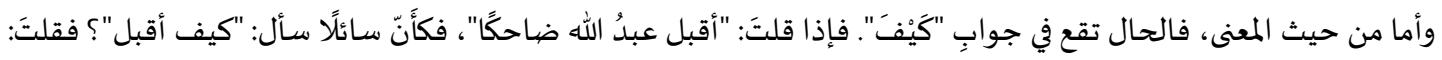

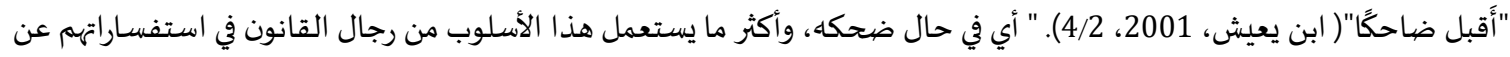
حال الجاني أو المجني وقت وقوع الحدث.

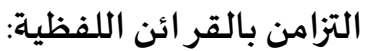

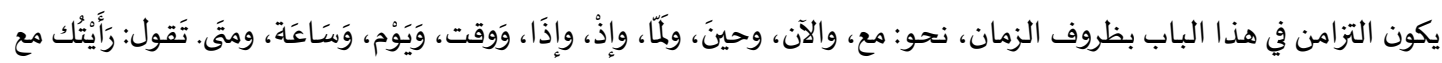

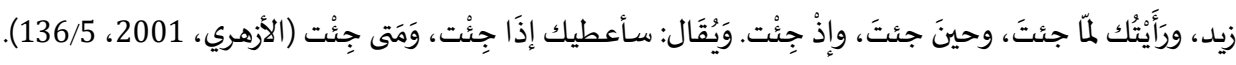
وكذلك يجمع الحروف التي تفيد المصاحبة كواو الحال، و واو المصاحبة بين حدثين في زمن واحتد إندا. 


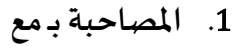

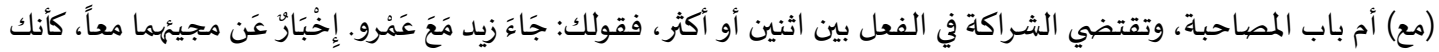

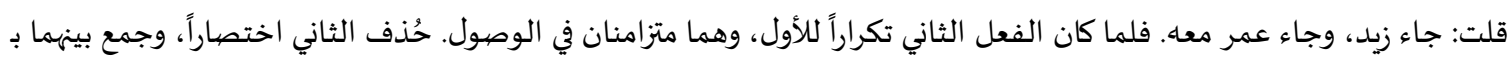

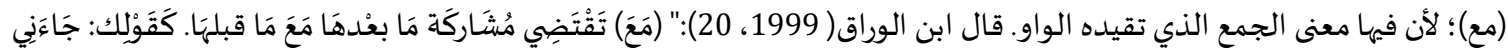

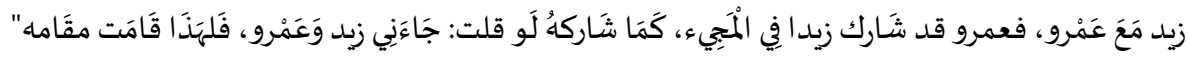

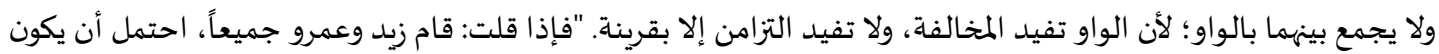

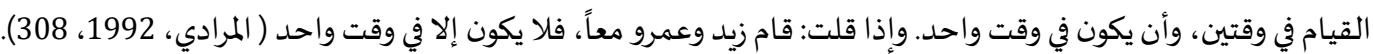

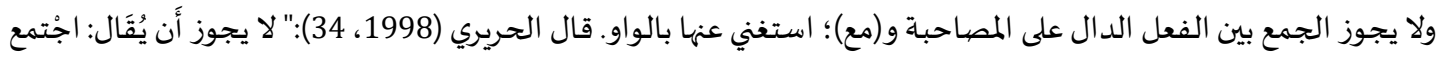

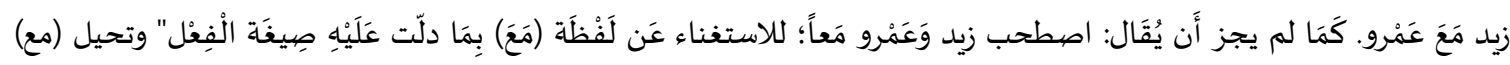

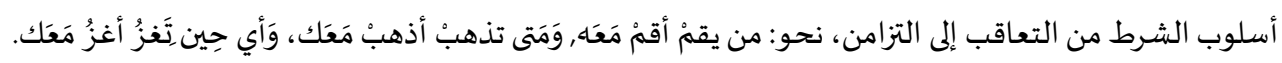

2. 2. ت واو المصياحبة

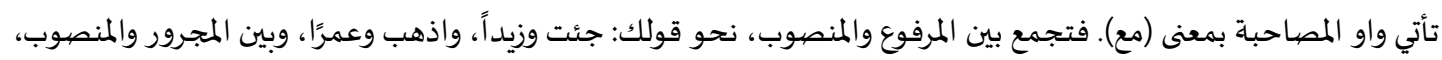

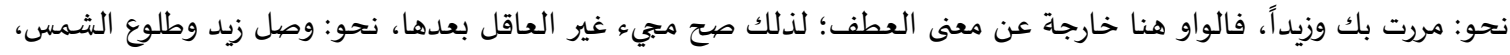

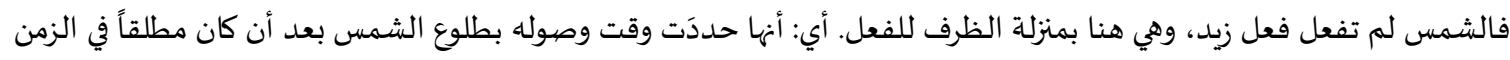
الماضي. وكذلك: سرت والجبل، حددت مكان سيره، فهي أفعال مقرونة بزمان أو مكان، وكأن المتحدث جعل الشمس والجبل ظرفين

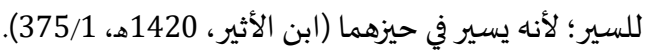

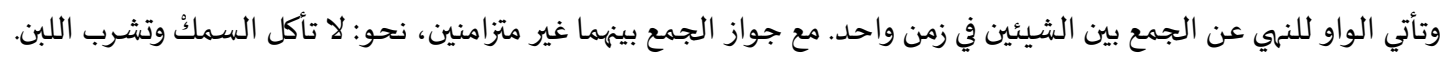

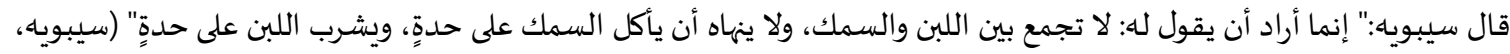

(43/3، 1988 3.

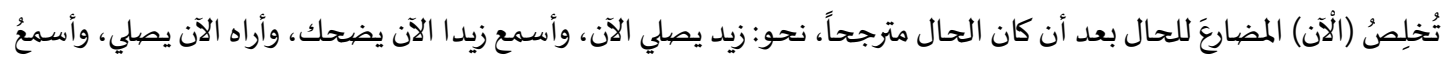

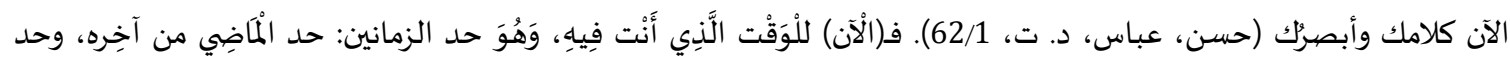

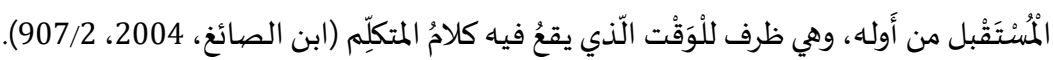

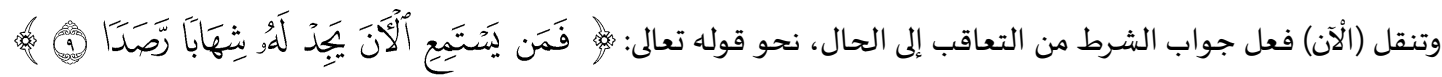
(سورة الجن: 9). (سعل العقوبة متزامنة مع الفعل. 4.

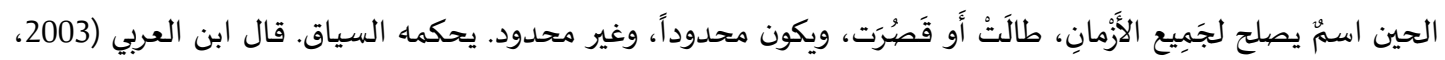

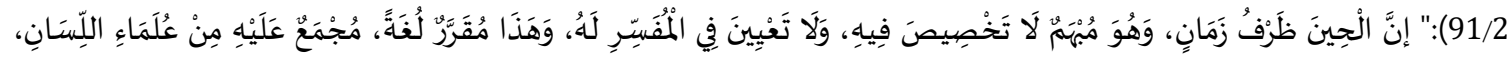

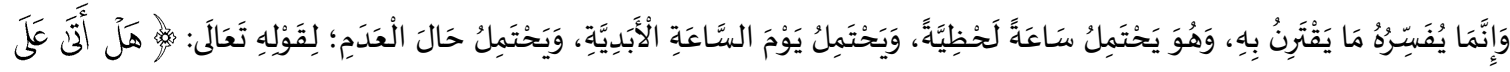

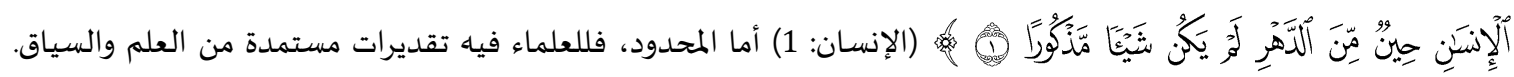

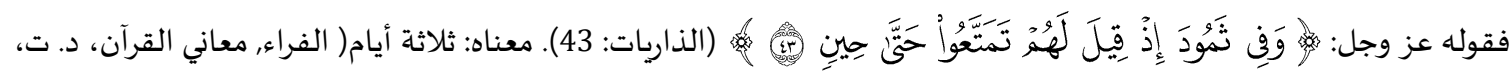

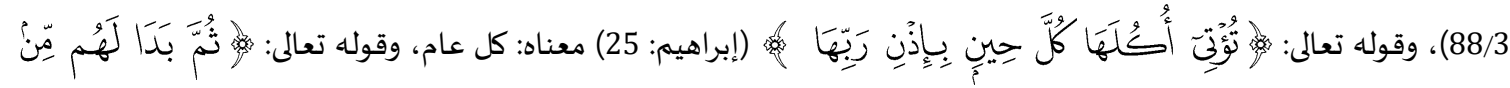

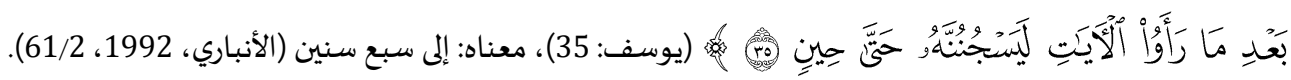

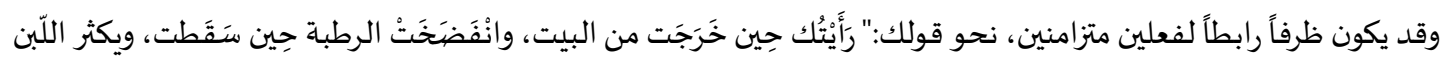

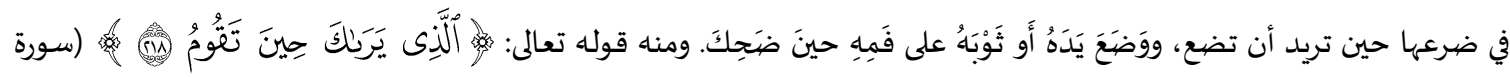

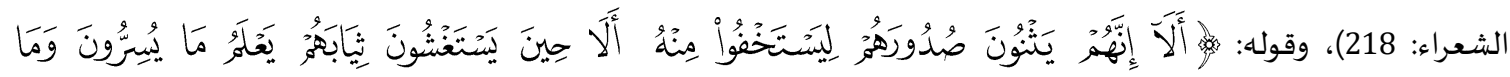




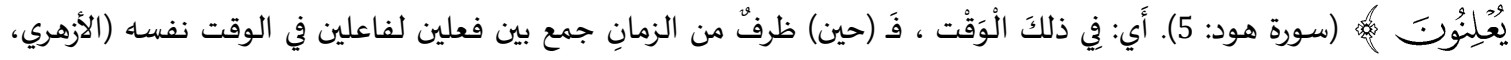

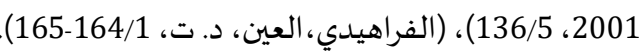

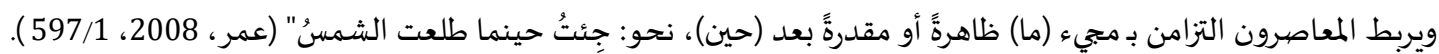

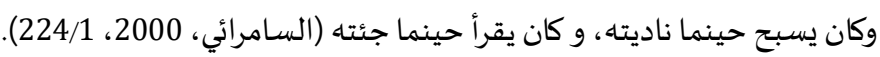

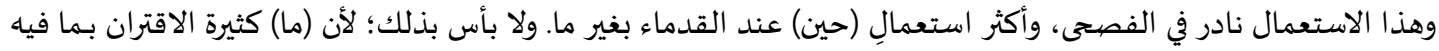
معنى الثرط.

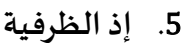

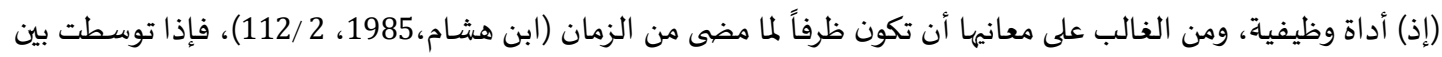

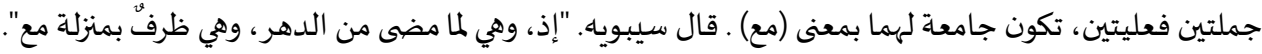

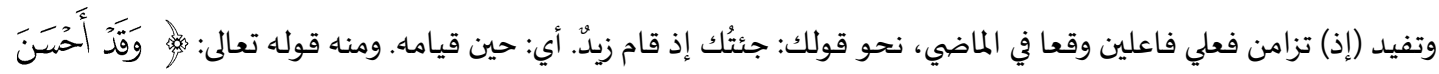

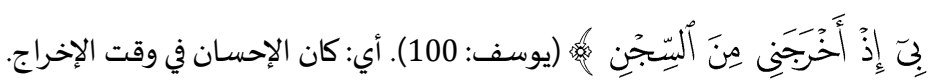

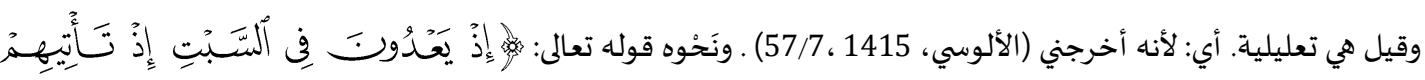

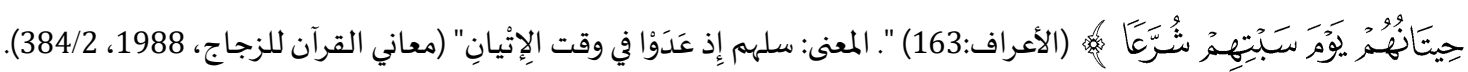

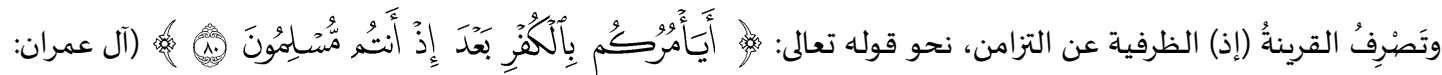
60) - 80 الظرف (بعد) دالّ هنا على التعاقب.

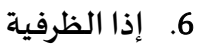

(إذًا) ظرف ميهم بمعنى حين. وتفيد التزامن المطلق في المستقبل بين جملتيها إذا أفادت الشرط؛ لأن الشرط على معنى المستقبل،

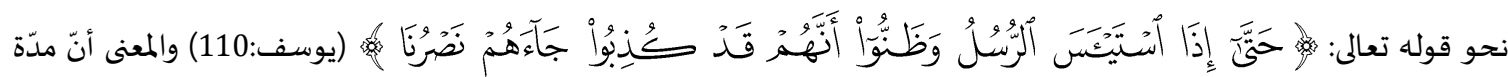
التكذيب والعداوة من الكفار وانتظار النصر من الله وتأميله قد تطاولت علهيهم وتمادت، حتى استشعروا القنوط وتوهموا أن لا نصر

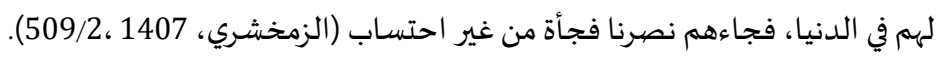

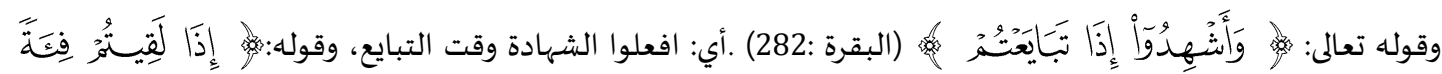

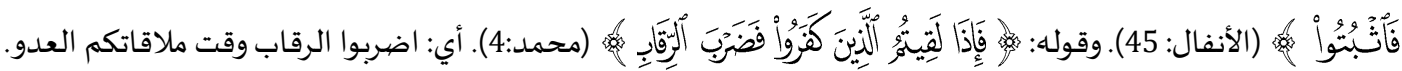

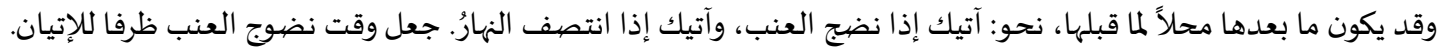

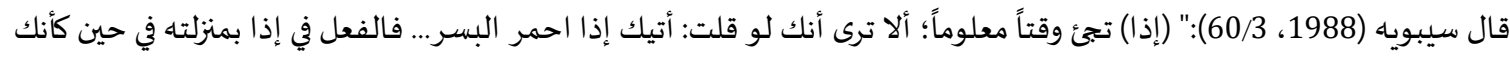

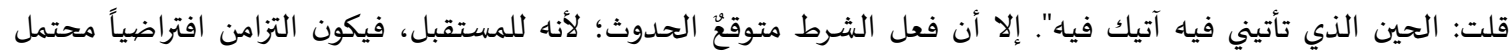

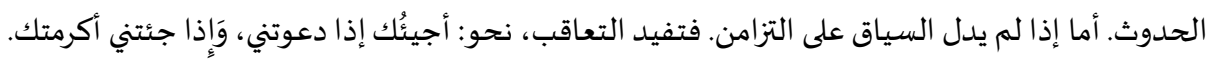

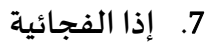

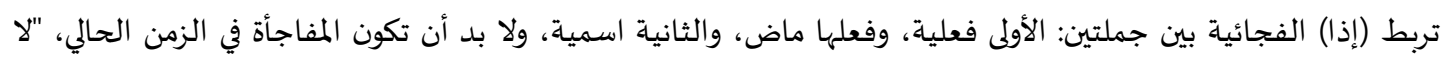

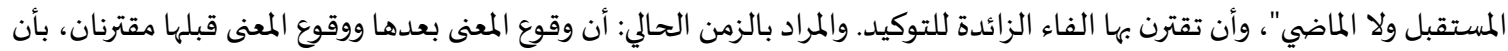

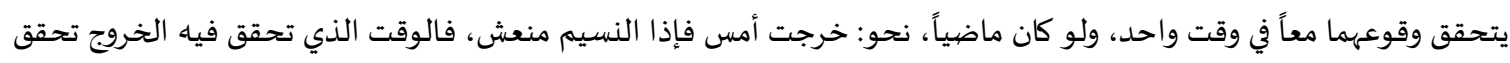

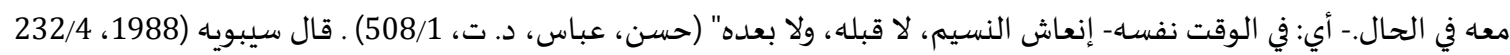

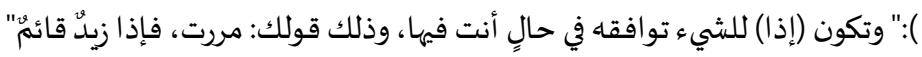

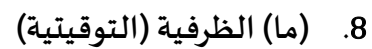

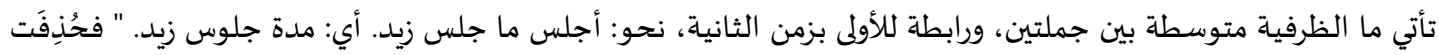

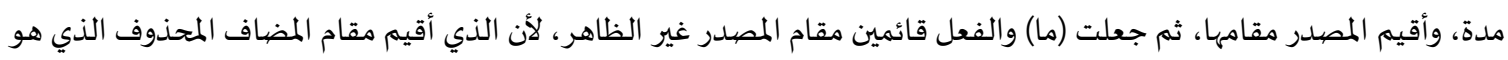

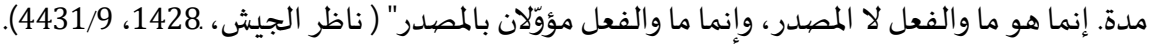


(لمام) مركبة من لم، وما الظرفية التوقيتية (العكبري، 1995، 48/2 )، ولها استعمالان: الأول أن تأتي ظرفية غير الشرطية، فتكون

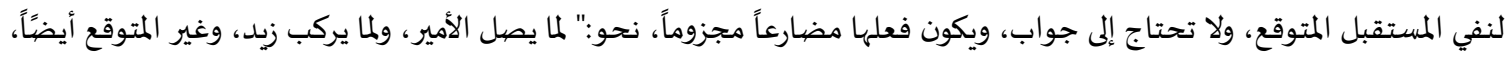

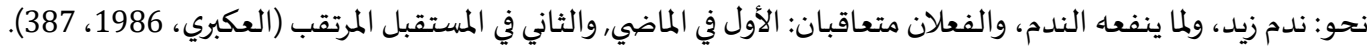

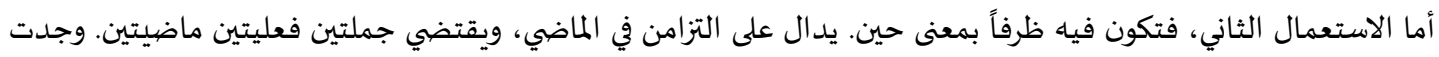

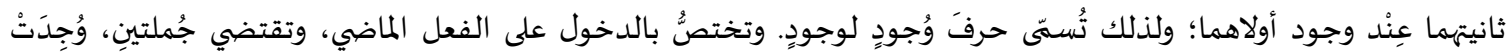

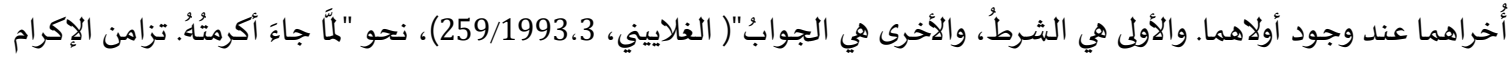

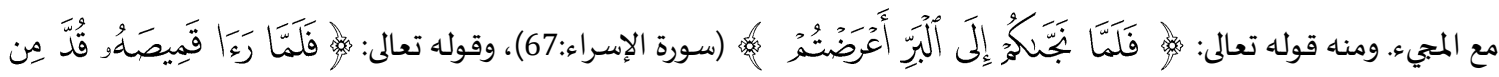

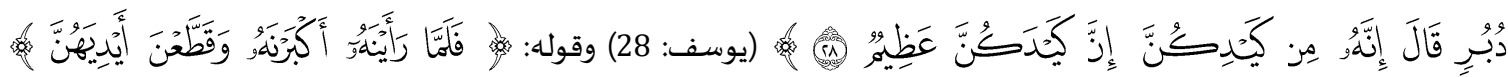
(يوسف: 31).

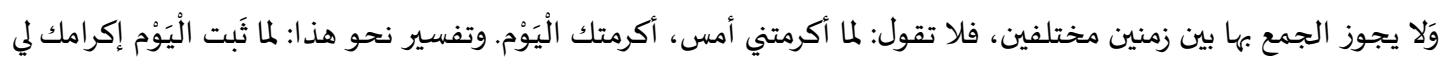

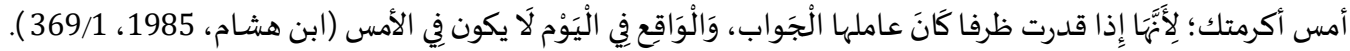

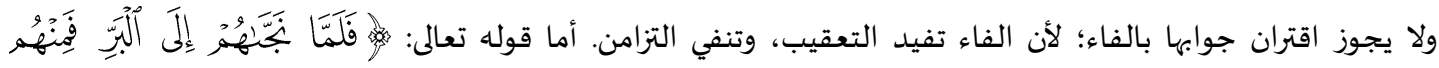

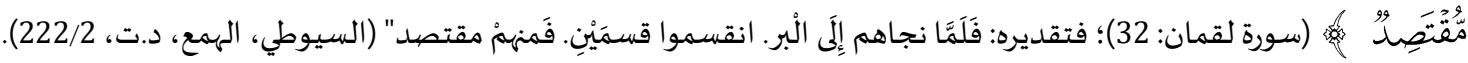
ولا يجوز اقتران جوابها بالفعل المضارع؛ ولذلك فسر قوله تعالى

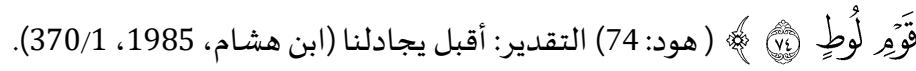

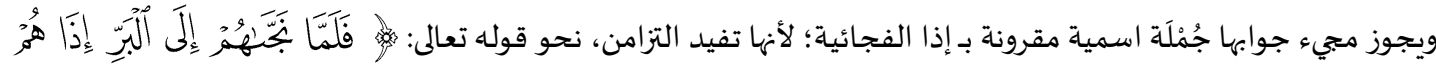

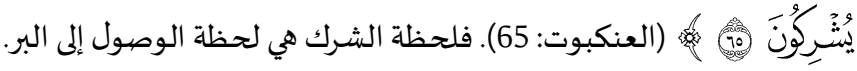

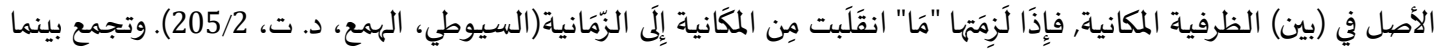

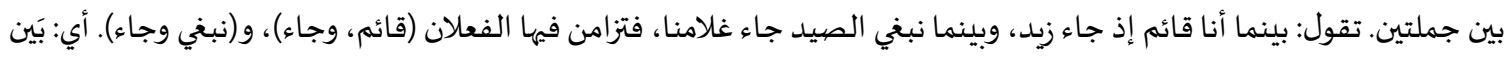

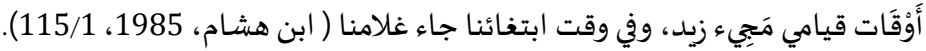

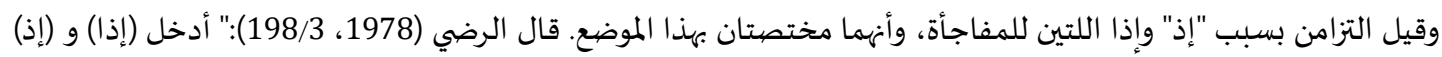

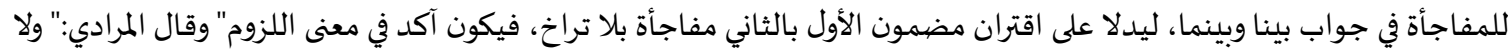

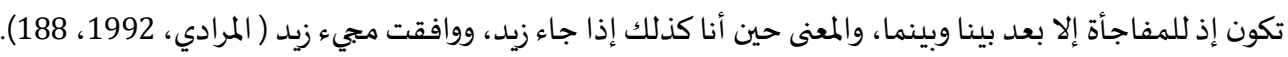

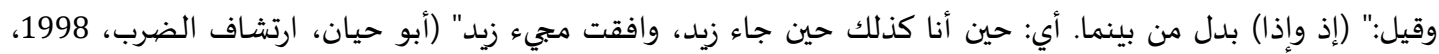

.$(1404 / 3$

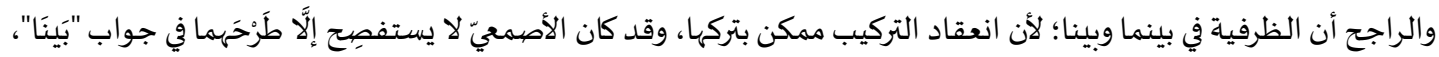

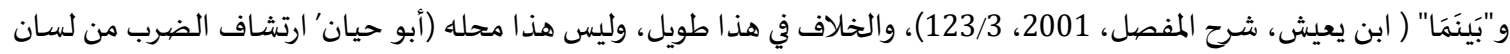

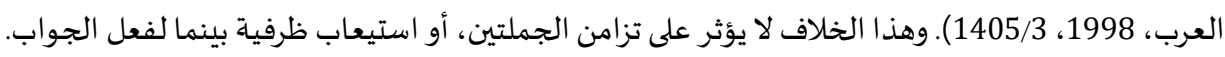

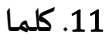

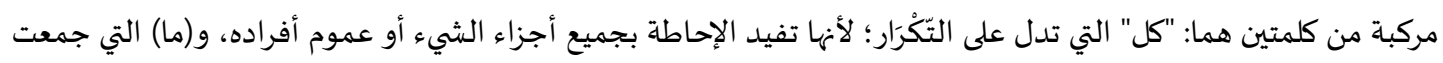

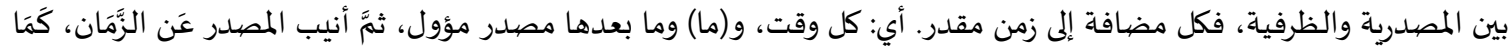

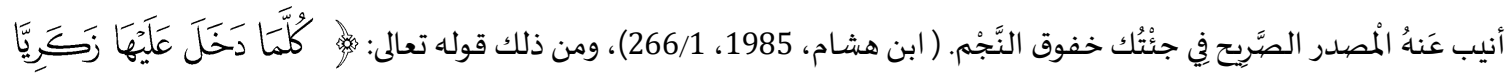

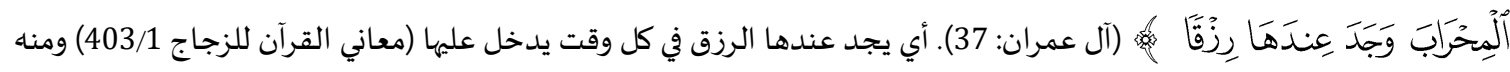

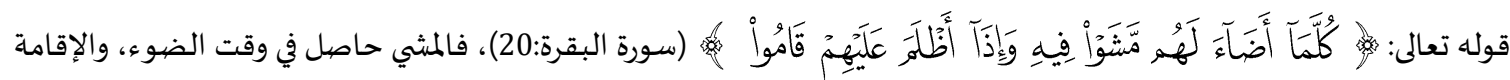




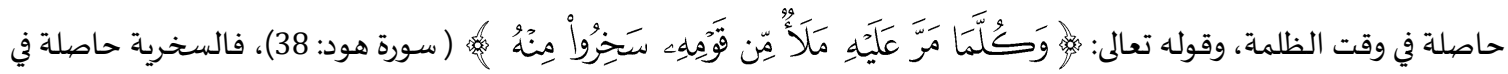
وقت المرور.

وقد يحتمل جواب كلما التعاقب إذا كان المعنى على المستقبل، نحو: كلما تأتيني آتيك. فوقت إتيان الأول غير وقت إتيان الثاني

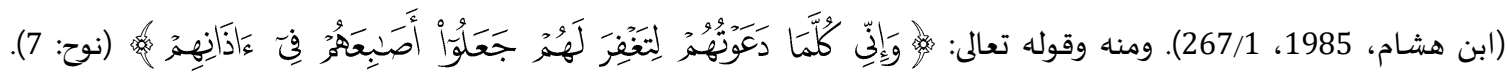
فالدعاء سابق لوضع الأصابع في الآذان.

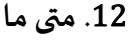

هي عند النحاة أداة شرط، وما زائدة، نحو: متى ما تأتنني آتِك"، ومتى ما تخرج أخرج، " أي: "متى ما تكن أكن": وفي أي وقت تكن

أكن،"( الأنباري، 2003، (529/2)

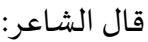

متى ما تلقني فردين ترجف ... رو انف أليتيك وتستطارا (البيت لعنترة بن شداد في ديو انه 234) فقد جاءت (متى ما) للربط بين جملتين في ظرف زماني واحد. كما كانت أينما للجمع في المكان. إلا أن أينما محل للأعلام، ومتى دئى محل للأفعال.

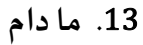

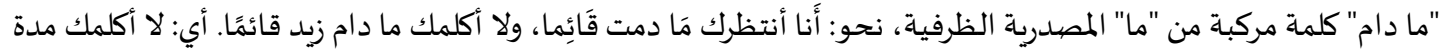

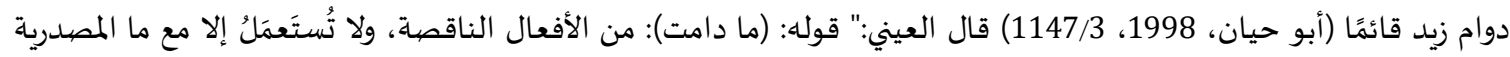

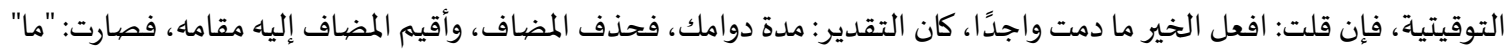

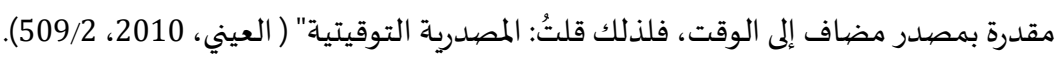

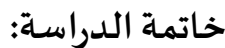

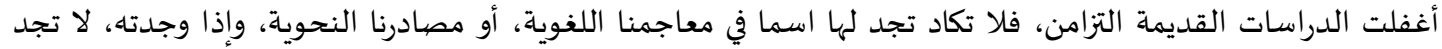

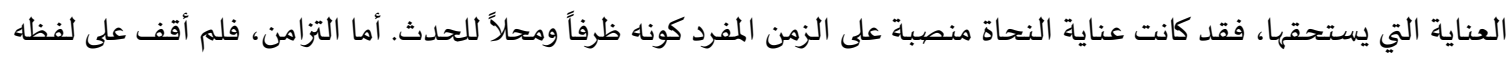

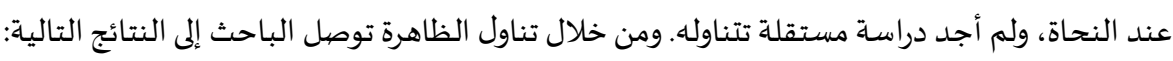
التزامن وقوع حدثين في الوقت نفسه، أو حدثين متصلين بلا انقطاع في زمن متصلي. هاء التزامن جامعاً بين فعلين ماضيين، أو فعلين مضارعين، أو فعلين مستقبليين.

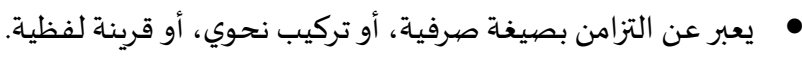

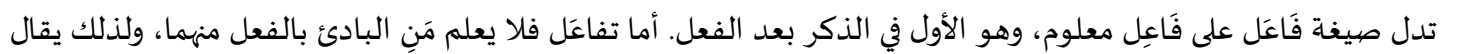

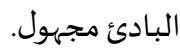
• • راعى التزامن تعدد معاني الأفعال، فكان بين فعلين لحاستين، أو فعل حاسة وفعل علة، أو فعل قلبي وفعل جارحة.

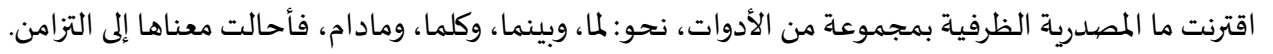

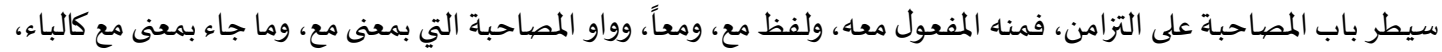
وفي، وعلى. • إذا كان جواب الشرط مجزوماً، فالفعلان متصلان. يعقب الثاني فهيما الأول، وإذا كان الجواب بـ إذا الفجائية، فالفعلان متزامنان في المستقبل المتوقع، وإذا كان الجواب بالفاء، فالجواب على التعقيب. هاءت اللغة وظيفية، فدلت على التزامن بين فعلين في الوقت نفسها، أو التفريق بينهما في الوقت نفساه.

$$
\text { • المراجع: القرآن الكريم }
$$

1. ابن الأثير، المبارك بن محمد (1420 هـ). البديع في علم العببية. تحقيق: فتحي أحمد علي الدين. الطبعة الأولى. المملكة العربية

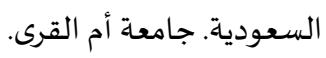


2. الأزهري، محمد بن أحمد (2001).تهنيب اللغة. تحقيق: محمد عوض مرعب. الطبعة الأولى. بيروت، دار إحياء التراث العربي.

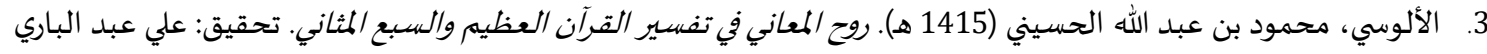
عطية. الطبعة الأولى. بيروت، دار الكتب العلمية. 4. الأنباري، عبد الرحمن بن محمد(1999).أسرار العببية. الطبعة الأولى. دار الأرقم بن أبي الأرقم.

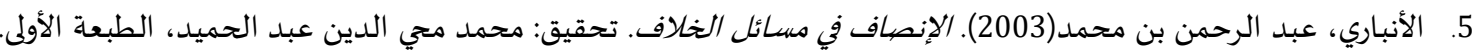
المكتبة العصبرية. 6. الأنباري، محمد بن القاسم (1992). الزاهر في معاني كلمات الناس. تحقيق: حاتم صالح الضامن، الطبعة الأولى. بيروت، مؤسسة الرسالة.

7. التهانوي، محمد بن علي (1996). موسوعة كشاف اصهطلاحات الفنون والعلوم. تحقيق: علي دحروج. نقل النص الفارسي إلى العربية: عبد الله الخالدي. ترجمة: جورج زيناني. الطبعة الأولى. بيروت- مكتبة لبنان. موسوان. 8. الثعالبي، عبد الملك بن محمد (2002). فقه اللغة وسر العربية. تحقيق: عبد الرزاق المهدي. الطبعة الأولى. إحياء التراث العربي. 9 9. ابن جني، أبو الفتح عثمان (د. ت ت). الخصيائص. الطبعة الرابعة. الهيئة المصبرية العامة للكتاب. 10. الجوهري، إسماعيل بن حماد (1978). الصحاح تاج اللغة وصحاح العببية. تحقيق: أحمد عبد الغفور عطار. الطبعة الرابعة. بيروت، دار العلم للملايين.

11. الحريري، القاسم بن علي (1998). درة الغواص. دحقيق: عرفات مطرجي. الطبعة الأولى. بيروت، مؤسسة الكتب الثقافية.

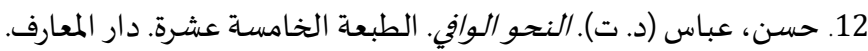
13. أبو حيان، محمد بن يوسف (1998). ارتشاف الضيرب من لسان العرب. تحقيق: رجب عثمان محمد. مراجعة: رمضان عبد العان

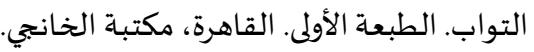

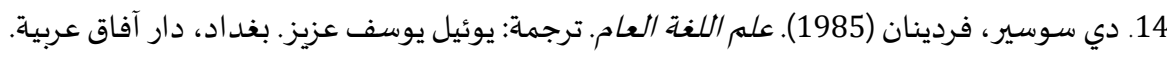

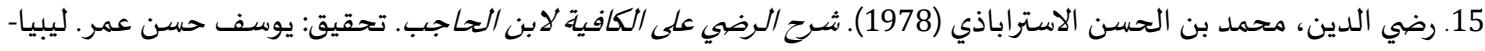
جامعة قار يونس. 16. الزجاج، أبو إسحاق، إبراهيم بن السري بن سهل (1988) معاني القرآن وإعرابه. تحقيق: عبد الجليل عبده شلبي. الطبعة الأولى. بيروت -عالم الكتب.

17. الزَّجَّاجي، أبو القاسم (1986). الإيضياح في علل النحو. تحقيق: مازن المبارك. الطبعة: الخامسـة. بيروت، لبنان، دار النفائس. 18. الزجاجي، عبد الرحمن بن إسحاق (1984). حروف المعاني والصفات. تحقيق: علي توفيق الحمد. الطبعة الأولى، بيروت، دأل مؤسسة الرسالة.

19. الزمخشري، محمود بن عمرو (1407هـ). الكشاف عن حقائق غوامض التننيل. الطبعة الثالثة. بيروت. دار الكتاب العربي. 20. السامرائي، فاضل صالح (2000). معاني النحو. الطبعة الأولى. الأردن، دار الفودو الفكر.

21. ابن السراج، محمد بن السري (د. ت). الأصيول في النحو. تحقيق: عبد الحسين الفتلي. بيروت، لبنان، مؤسسة الرسالة. 22. سيبويه، عمرو بن عثمان (1988). الكتاب. تحقيق: عبد السلام محمد هارون. الطبعة الثالثة. القاهرة، مكتبة الخانجي. 23. السيرافي، أبو سعيد الحسن بن عبد الله بن المرزبان (2008). شرح كتاب سيبويه. تحقيق: أحمد حسن مهلدي، علئ علي سيد علي. الطبعة الأولى. بيروت - لبنان، دار الكتب العلمياة.

24. السيوطي، عبد الرحمن بن أبي بكر (د. ت). همع الهوامع. تحقيق: عبد الحميد هنداوي. مصر، المكتبة المباء التوفيقية.

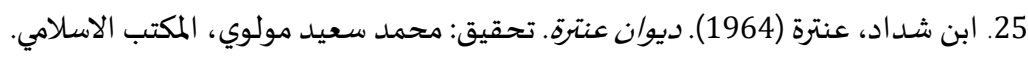
26. ابن الصائغ، محمد بن حسن بن سِباع (2004). اللمحة في شـح الملحة . تحقيق: إبراهيم بن سالم الصياعدي. الطبعة الأولى،

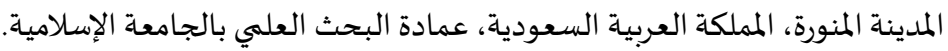
27. ابن العربي، أبو بكر المعافري الاشبيلي (2003). أحكام القرآن. مراجعة: محمد عبد المقادر عطا. الطبعة الثالثة، بيروت - لبنان،

$$
\text { دار الكتب العلمية. }
$$
28. ابن عقيل، عبد الله بن عبد الرحمن (1980). شرح /بن عقيل. تحقيق: محمد محيب الدين عبد الحميد. الطبعة العشرون، القاهرة، دار التراث. 
29. العكبري، عبد الله بن الحسين (1986). التبيين عن مذاهب النحويين. تحقيق: عبد الرحمن العثيمين. الطبعة الأولى، دار الغرب الإسلامي.

30. العكبري، عبد الله بن الحسين (1995). اللباب في علل البناء والإعراب. تحقيق: عبد الإله النهان. الطبعة الأولى. دمشق، دار الفكر. 31. عمر، أحمد مختار عبد الحميد (2008). معجمم اللغة العببية المعاصرة. الطبعة الأولى. عالم الكتب.

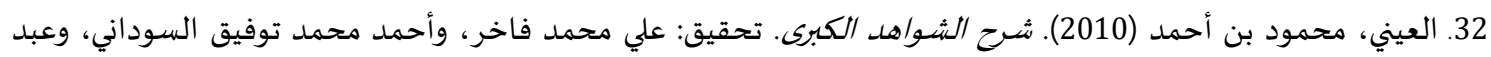

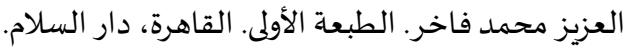
33. الفلايينى، مصطفى بن محمد سليم (1993). جامع الدروس العبرية. الطبعة الثامنة والعشرون. صيدا، بيروت، المكتبة العبدا العصرية. 34. الفراء، يحيى بن زياد (د. ت). معاني القرآن. تحقيق: أحمد يوسف النجاتي. و محمد علي النجار وعبد الفتاح إسماعيل الشلبي.

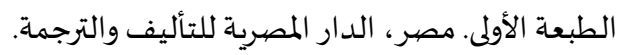

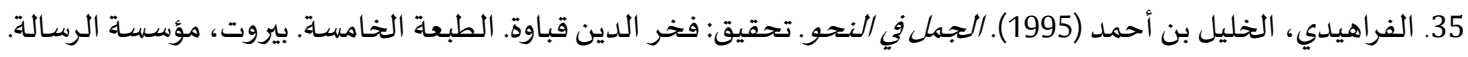

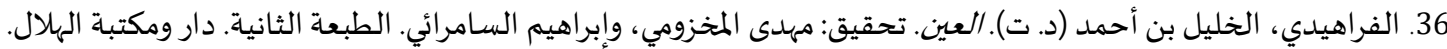

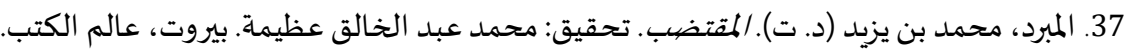

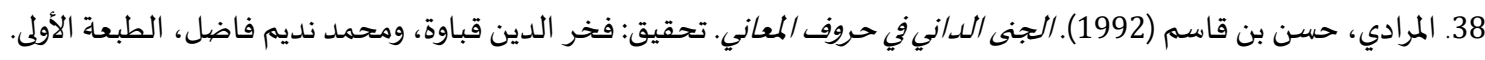
بيروت. لبنان. دار الكتب العلمية.

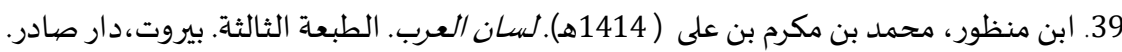
40. ناظر الجيش، محمد بن يوسف (1428هـ). تمهيد القواعد بشرح تسهيل الفوائد. دراسة وتحقيق: علي محمد فاخر وآخرون. الطبعة الأولى. القاهرة، دار السلام. 41. ابن هشام، عبد الله بن يوسف (1985). مغني اللبيب. تحقيق: مازن المبارك، ومحمد علي حمد الله، الطبعة السادسة. دمشق، دار الفكر. 42. ابن الوراق، محمد بن عبد الله (1999). علل النحو. تحقيق: محمود جاسم محمد الدرويش. الطبعة الأولى. الرياض، السعودية، مكتبة الرشد. 43. ابن يعيش، محمد بن علي (2001). شحح المفصل للزمخشري. تحقيق: إميل بديع يعقوب. الطبعة الأولى. بيروت، لبنان، دار الكتب العلمية. 
المجلة الدولية للدراسـات اللغوبة والأدبية العربية

International Journal for Arabic Linguistics and Literature Studies (JALLS)

\title{
Synchronicity in Arabic language (semantic study)
}

\author{
Fouad Hamada \\ Department of Arabic Language, Faculty of Arts, Al-Quds Open University, Palestine \\ foadhamada@gmail.com
}

Received : 18/5/2021 Revised: 7/6/2021 Accepted : 19/6/2021 DOI : https://doi.org/10.31559/JALLS2021.3.2.3

Abstract: Synchronicity has been frequently used in modern sciences, both linguistically and idiomatically, which necessitated ingraining its meaning and the manner it is used. As for our ancient linguistic dictionaries and grammatical resources, synchronicity has been neglected, and the grammarians confined themselves with dealing with the verb tense and associating it with the adverb as the subject of the event.

The importance of this research stems from the need for the eloquent use, the pursuit of linguistic correctness so that the pronouncement of the word matches its meaning, knowing the language structures that express the intention and to fulfill the purpose as well.

This study aims to identify the lexical items, structures, expressions and linguistic methods used by Arabs to link two events that occurred in one period, besides taking advantage of them in a contemporary use to ensure the integrity of the language.

The researcher adopted the descriptive besides the inductive approach, whereby the researcher followed the linguistic evidence from the Holy Qur'an and words of Arabs that were used in their prose and poetry. He also attempted to extract the rule to use synchronicity both linguistically and idiomatically. The researcher concluded that synchronicity is branch over tense is in the past, present, or future, which combines two events occurring at the same time, or between two concurrent events, as it is expressed in a morphological form, a grammatical structure, or a verbal context.

Keywords: Synchronicity; two events; concurrent; morphological form; grammatical structure; verbal context.

\section{References:}

1. Abn 'qyl, 'bd Allh Bn 'bd Alrhmn (1980). Shrh Abn 'qyl. Thqyq: Mhmd Mhyy Aldyn 'bd Alhmyd. Altb'h Al'shrwn, Alqahrh, Dar Altrath.

2. Al'kbry, 'bd Allh Bn Alhsyn (1986). Altbyyn 'n Mdahb Alnhwyyn. Thqyq: 'bd Alrhmn Al'thymyn. Altb'h Alawla, Dar Alghrb Aleslamy.

3. Al'kbry, 'bd Allh Bn Alhsyn (1995). Allbab Fy 'll Albna' Wale'rab. Thqyq: 'bd Alelh Alnbhan. Altb'h Alawla. Dmshq, Dar Alfkr.

4. 'mr, Ahmd Mkhtar 'bd Alhmyd (2008). M'jm Allghh Al'rbyh Alm'asrh. Altb'h Alawla. 'alm Alktb.

5. Abn Al'rby, Abw Bkr Alm'afry Alashbyly (2003). Ahkam Alqran. Mraj'h: Mhmd 'bd Alqadr 'ta. Altb'h Althalthh, Byrwt - Lbnan, Dar Alktb Al'lmyh.

6. Al'yny, Mhmwd Bn Ahmd (2010). Shrh Alshwahd Alkbra. Thqyq: 'ly Mhmd Fakhr, Wahmd Mhmd Twfyq Alswdany, W'bd Al'zyz Mhmd Fakhr. Altb'h Alawla. Alqahrh, Dar Alslam.

7. Alalwsy, Mhmwd Bn 'bd Allh Alhsyny (1415 H). Rwh Alm'any Fy Tfsyr Alqran Al'zym Walsb' Almthany. Thqyq: 'ly 'bd Albary 'tyh. Altb'h Alawla. Byrwt, Dar Alktb Al'lmyh.

8. Alanbary, 'bd Alrhmn Bn Mhmd (1999). Asrar Al'rbyh. Altb'h Alawla. Dar Alarqm Bn Aby Alarqm. 
9. Alanbary, 'bd Alrhmn Bn Mhmd (2003). Alensaf Fy Msa'l Alkhlaf. Thqyq: Mhmd Mhy Aldyn 'bd Alhmyd, Altb'h Alawla. Almktbh Al'esryh.

10. Alanbary, Mhmd Bn Alqasm (1992). Alzahr Fy M'any Klmat Alnas. Thqyq: Hatm Salh Aldamn, Altb'h Alawla. Byrwt, M'sst Alrsalh.

11. Abn Alathyr, Almbark Bn Mhmd (1420 H). Albdy' Fy 'lm Al'rbyh. Thqyq: Fthy Ahmd 'ly Aldyn. Altb'h Alawla. Almmlkh Al'rbyh Als'wdyh. Jam't Am Alqra.

12. Alazhry, Mhmd Bn Ahmd (2001). Thdyb Allghh. Thqyq: Mhmd 'wd Mr'b. Altb'h Alawla. Byrwt, Dar Ehya' Altrath Al'rby.

13. Dy Swsyr, Frdynan (1985). 'Im Allghh Al'am. Trjmh: Yw'yl Ywsf 'zyz. Bghdad, Dar Afaq 'rbyh.

14. Alfra', Yhya Bn Zyad (D. T). M'any Alqran. Thqyq: Ahmd Ywsf Alnjaty. W Mhmd 'ly Alnjar W'bd Alftah Esma'yl Alshlby. Altb'h Alawla. Msr, Aldar Almsryh Lltalyf Waltrjmh.

15. Alfrahydy, Alkhlyl Bn Ahmd (1995). Aljml Fy Alnhw. Thqyq: Fkhr Aldyn Qbawh. Altb'h Alkhamsh. Byrwt, M'sst Alrsalh.

16. Alfrahydy, Alkhlyl Bn Ahmd (D. T). Al'yn. Thqyq: Mhda Almkhzwmy, Webrahym Alsamra'y. Altb'h Althanyh. Dar Wmktbh Alhlal.

17. Alghlayyna, Mstfa Bn Mhmd Slym (1993). Jam' Aldrws Al'rbyh. Altb'h Althamnh Wal'eshrwn. Syda, Byrwt, Almktbh Al'sryh.

18. Alhryry, Alqasm Bn 'ly (1998). Drt Alghwas. Thqyq: 'rfat Mtrjy. Altb'h Alawla. Byrwt, M'sst Alktb Althqafyh.

19. Abn Hsham, 'bd Allh Bn Ywsf (1985). Mghny Allbyb. Thqyq: Mazn Almbark, Wmhmd 'ly Hmd Allh, Altb'h Alsadsh. Dmshq, Dar Alfkr.

20. Hsn, 'bas (D. T). Alnhw Alwafy. Altb'h Alkhamsh 'shrh. Dar Alm'arf.

21. Abw Hyan, Mhmd Bn Ywsf (1998). Artshaf Aldrb Mn Lsan Al'rb. Thqyq: Rjb 'thman Mhmd. Mraj'h: Rmdan 'bd Altwab. Altb'h Alawla. Alqahrh, Mktbt Alkhanjy.

22. Abn Jny, Abw Alfth 'thman (D. T). Alkhsa's. Altb'h Alrab'h. Alhy'h Almsryh Al'amh Llktab.

23. Althanwy, Mhmd Bn 'ly (1996). Mwsw't Kshaf Astlahat Alfnwn Wal'lwm. Thqyq: 'ly Dhrwj. Nql Alns Alfarsy Ela Al'rbyh: 'bd Allh Alkhaldy. Trjmt: Jwrj Zynany. Altb'h Alawla. Byrwt- Mktbt Lbnan.

24. Alth'alby, 'bd Almlk Bn Mhmd (2002). Fqh Allghh Wsr Al'rbyh. Thqyq: 'bd Alrzaq Almhdy. Altb'h Alawla. Ehya' Altrath Al'rby.

25. Aljwhry, Esma'yl Bn Hmad (1978). Alshah Taj Allghh Wshah Al'rbyh. Thqyq: Ahmd 'bd Alghfwr 'tar. Altb'h Alrab'h. Byrwt, Dar Al'Im Llmlayyn.

26. Almbrd, Mhmd Bn Yzyd (D. T). Almqtdb. Thqyq: Mhmd 'bd Alkhalq 'zymh. Byrwt, 'alm Alktb.

27. Almrady, Hsn Bn Qasm (1992). Aljna Aldany Fy Hrwf Alm'any. Thqyq: Fkhr Aldyn Qbawh, Wmhmd Ndym Fadl, Altb'h Alawla. Byrwt. Lbnan. Dar Alktb Al'lmyh.

28. Abn Mnzwr, Mhmd Bn Mkrm Bn 'Ela (1414h). Lsan Al'rb. Altb'h Althalthh. Byrwt,Dar Sadr.

29. Nazr Aljysh, Mhmd Bn Ywsf (1428h). Tmhyd Alqwa'd Bshrh Tshyl Alfwa'd. Drash Wthqyq: 'ly Mhmd Fakhr Wakhrwn. Altb'h Alawla. Alqahrh, Dar Alslam.

30. Rdy Aldyn, Mhmd Bn Alhsn Alastrabady (1978). Shrh Alrdy 'la Alkafyh Labn Alhajb. Thqyq: Ywsf Hsn 'mr. Lybya- Jam't Qar Ywns.

31. Alzjaj, Abw Eshaq, Ebrahym Bn Alsry Bn Shl (1988) M'any Alqran We'erabh. Thqyq: 'bd Aljlyl 'bdh Shlby. Altb'h Alawla. Byrwt -'alm Alktb.

32. Alzjajay, Abw Alqasm (1986). Aleydah Fy 'll Alnhw. Thqyq: Mazn Almbark. Altb'h: Alkhamsh. Byrwt, Lbnan, Dar Alnfa's.

33. Alzjajy, 'bd Alrhmn Bn Eshaq (1984). Hrwf Alm'any Walsfat. Thqyq: 'ly Twfyq Alhmd. Altb'h Alawla, Byrwt, M'sst Alrsalh.

34. Alzmkhshry, Mhmwd Bn 'mrw (1407h). Alkshaf 'n Hqa'q Ghwamd Altnzyl. Altb'h Althalthh. Byrwt. Dar Alktab Al'rby.

35. Abn Alsa'gh, Mhmd Bn Hsn Bn Sba' (2004). Allmhh Fy Shrh Almlhh. Thqyq: Ebrahym Bn Salm Alsa'dy. Altb'h Alawla, Almdynh Almnwrh, Almmlkh Al'rbyh Als'wdyh, 'madh Albhth Al'lmy Baljam'h Aleslamyh.

36. Alsamra'y, Fadl Salh (2000). M'any Alnhw. Altb'h Alawla. Alardn, Dar Alfkr. 
37. Abn Shdad, 'ntrh (1964). Dywan 'ntrh. Thqyq: Mhmd S'yd Mwlwy, Almktb Alaslamy.

38. Abn Alsraj, Mhmd Bn Alsry (D. T). Alaswl Fy Alnhw. Thqyq: 'bd Alhsyn Alftly. Byrwt, Lbnan, M'sst Alrsalh.

39. Sybwyh, 'mrw Bn 'thman (1988). Alktab. Thqyq: 'bd Alslam Mhmd Harwn. Altb'h Althalthh. Alqahrh,Mktbh Alkhanjy.

40. Alsyrafy, Abw S'yd Alhsn Bn 'Ebd Allh Bn Almrzban (2008). Shrh Ktab Sybwyh. Thqyq: Ahmd Hsn Mhdly, 'ly Syd 'ly. Altb'h Alawla. Byrwt - Lbnan, Dar Alktb Al'lmyh.

41. Alsywty, 'bd Alrhmn Bn Aby Bkr (D. T). Hm' Alhwam'. Thqyq: 'bd Alhmyd Hndawy. Msr, Almktbh Altwfyqyh.

42. Abn Alwraq, Mhmd Bn 'bd Allh (1999). 'll Alnhw. Thqyq: Mhmwd Jasm Mhmd Aldrwysh. Altb'h Alawla. Alryad, Als'wdyh, Mktbt Alrshd.

43. Abn Y'ysh, Mhmd Bn 'ly (2001). Shrh Almfsl Llzmkhshry. Thqyq: Emyl Bdy' Y'qwb. Altb'h Alawla. Byrwt, Lbnan, Dar Alktb Al'lmyh. 